AN INVESTIGATION OF THE VERTICAL VARIATION OF MIEHT SCATTERING IN MONTEREY BAY

CHARLES H. BASSETT

and

HARRY C. FURMINGER 
DUDLEY KNOX LIBRARY

NAVALPCSTIT

MONTEREY IA 50,405101 
: 


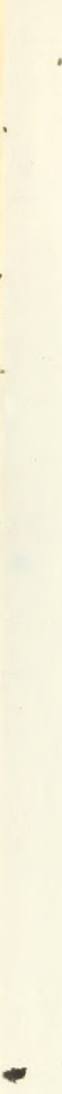




\title{
AN INVESTIGATION OF THE \\ VERTICAL VARIATION OF LIGHT SCATTERING \\ IN MONTEREY BAY, CALIFORNIA
}

\author{
$* * * * *$ \\ Charles H. Bassett, Jr. \\ and \\ llopry C, Furminor
}




\title{
AN INVESTIGATION OF THE \\ VERTICAL VARIATION OF LIGHT SCATTERING \\ IN MONTEREY BAY, CALIFORNIA
}

\author{
by \\ Charles H. Bassett, Jr. \\ Lieutenant, United States Navy \\ and \\ Harry C. Furminger \\ Lieutenant, United States Navy
}

Submitted in partial fulfillment of

the requirements for the degree of

MASTER OF SCIENCE

United States Naval Postgraduate School

Monterey, California

1965 
$1<=5$

IUPS APQ HIE

1965

BASSETT,C 
AN INVESTIGATION OF THE

VERTICAL VARIATION OF LIGHT SCATTERING

IN MONTEREY BAY, CALIFORNIA

$$
\text { by }
$$

Charles H. Bassett, Jr.

$$
\text { and }
$$

Harry C. Furminger

Th1s work is accepted as fulfiling the thesis requirements for the degree of MASTER OF SCIENCE

$$
\text { from the }
$$

United states Naval Postgraduate School 



\section{ABSTRACT}

An investigation of the vertical variations of the scattering coefficients for $\nabla 1$ sible light at a selected location in Monterey Bay, California, was conducted during December, 1964, January and February, 1965. Forty-81x water samples were collected at varlous depths on five separate sampling days. Where possible, concurrent light attenuation (horizontal) and solar light extinction measurements were made in s1tu. The water samples collected were analyzed for density and inorganic phosphates.

From the scattering coefficients computed, and hand-f1tting of scattering runction curves with theoretical curves, the particle size and particle concentration was estimated for each sample.

The relationships between the sea water density, phosphate conitent, and the emplrically derived scattering coefflcient. particle size, and particle concentration were examined. The only significant correlation found is that between particle size and particle concentration. A particle concentration maximum was observed above and adjacent to the pycnocline where one existed. 

TABLE OF CONTENTS

Section

Title

Page

1. Introduction 1

1.1 Purpose 1

1.2 Theory 2

1.2.1 Light Attenuation 3

1.2.2 Light Absorption 4

1.2.3 Light Scattering 5

1.2.4 The Effect of Particle Size, Particle Concentration, and Wavelength on scatterlne ?

1.3 Station Oceanographic Climatology 9

2. Equipment 13

2.1 Sea Water Attenuation Hydrophotometer 13

2.2 Insolation Extinction Hydrophotogeter 14

2.3 Scattering Analys1s Apparatus 14

2.3.1 Constructed Laboratory Model 14

2.3.2 Aminco Light Scattering Micro-
photometer

2.4 Salinity Determination Apparatus 17

2.5 Phosphate Determination Apparatus 18

3. Procedure 19

3.1 Sample Collection and Attenuation Measurement

3.2 Forward Angle scattering Measurement 21

4. Data 23

4.1 Light Attenuation Data 23

4.2 Iight Extinction Data 23 



\section{TABLE OF CONTENTS}

Section

4.3 Scattering Coefficlent, Density, Phosphate, 24 Particle size and Concentration Data

5. Data Interpretation 26

6. Conclusions and Acknowledgements 32

Blbllography 35

Appendix

I Data Tables 38

II Illustrations 65 



\section{LIST OF TABLES}

Table

Page

1. Median Temperature and Salinity 38

2. Light Attenuation Data 39

3. Light Extinction Data 41

4. Scattering Coefricient, Density, Phosphate,
Particle Size and Concentration

5. Measured Scattering Intensity Data 49 



\section{LIST OF ILLUSTRATIONS}

Figure

Page

1. Light Energy Attenuation

2. Schematic Scatterometer

3. The Single Particle $K$ Field

4. Climatological Profiles

5. Relative Radiance With Depth

6. Soripps Alpha Meter

7. Extinction Hydrophotometer

8. Constructed Laboratory Scattering Photometer

9. Constructed Laboratory Scattering Photometer

10. Aminco Scattering Photometer

11. Monteres Bay, California

12. Single Particle scattering Intensity Curves, $m=1.33$

13. Single Particle Scattering Intensity Curves, $m=2.0,1.55,1.44$

14. Single Particle Scattering Intensity Curves, $m=1.20$

15. Measured Scattering Intensity Curves 80

16. Sample Profiles, 21 December, 1964

17. Sample Profiles, 6 January, 196582

18. Sample Profiles, 8 January, 1965

19. Sample Profiles, 26 January, 1965

20. Sample Profiles, 19 February, 1965

21. Graph of Scattering and Concentration 86

22. Scattering Field for Radius and Concentration 87

23. Scattering Field for Phosphate Content and Density 
1. Introduction

1.1 Purpose

In recent years, much research effort has been expended in the field of underwater optics, due primarily to the ract that many of the parameters in physical, biological, and chem1cal oceanography depend upon the physics associated with the transmission of light through sea water. Scientists are now investigating the areal distribution of the horizontal light attenuation as it varies with depth; (Hughes, R., at the Naval Ordnance Test Station, China Lake, California, personal communication); also under investigation is the areal varlation of light scattering [35]. The next logical step is a more detalled study of the vertical variation of light scattering; and its causes, effects, and methods of investigation. In this paper the approach to light scattering is through a study of the variation of light scatterers. From such a study, parameters involved in several oceanographic disciplines may be forecast, resulting in the application of research in underwater light transmission to areas such as:

1. development of an underwater coherent light source for use in detection, ranging and communication;

2. the use of attenuation coefficients or scatter coefficients as a means of typing water;

3. the general improvement of underwater photography and television techniques;

4. the study of the penetration of sea water by solar radiation and the resulting effect on productivity; 

5. the study of solar radiation penetration and its effect on sea surface temperature and ocean thermal structure.

Th1s investigation emphasizes the utility of scattering analysis as an integral part of descriptive oceanography. This study began in December, 1963, with field sampling beginning a year later. During this time, the authors investigated the oceanographic climatology of the Monterey Bay Area to determine the best sampling area. The sampling area finally chosen was selected considering proximity to shore, water depth, and avallability of past oceanographic data $[8,32]$. 



\subsection{Theory}

Before discussing the variation of scatterers, the applicable definitions and physical relationships for light transmission through water should be reviewed.

\subsubsection{Light Attenuation}

The attenuation of light is the diminution of intensity of a light beam during its passage through a medlum such as sea water, and it results from a number of processes. As shown by Tyler, et. al., this attenuation is mainly attributable to the scattering and absorbing properties of the medium [23].

The function expressing the attenuation of light transmission through a medium is

$$
N_{r}=N_{0} e^{-\alpha l}
$$

an expression of Beer's Law as interpreted by Tyler [II]. $N_{0}$ and $N_{r}$ are the source and receiver intensity, respectively, as measured by an "Alpha" (attenuation) meter. The range $l$ is the measured distance between the source and the recelver. A plot of various ranges compared with $\ln \left(\mathrm{Nr}_{\mathrm{r}} / \mathrm{N}_{0}\right)$ should give a straight line when plotted against the total attenuation coefficient of the light beam, $\alpha$.

The attenuation coefficient is primarily the sum of the total scattering coefficient, $S$, and the absorption coefficlent, $a$, or

$$
\alpha=2+s
$$



These coefficlents have the units of length ${ }^{-1}$.

In a medium such as sea water, the presence of contaminants and solutes alters the scattering and the absorption. In fact, any diatomic molecule of the medium or solute will be a source of possible attenuation [10].

\subsubsection{Light Absorption}

Scattering is a process which results in the redirection of light energy. Iight absorption, in contrast, is a process which is an onergy transformation. The absorbing substance diminishes the incident light energy by changing it into other forms of energy, such as heat energy and moleoular (rotational) energy [10].

The measurement of absorption coefficients may be complicated and sometimes measurement is performed by indirect means [22]. To measure absorption in a medium such as sea water, it is desirable to use a collimated light source to reduce scattering to minimum; the optimum source is a highly collimated laser beam. Because of the monochromatic properties of sea water, the usual approach is to use a narrow band sharp cutoff filter with a mercury arc lamp as a light source for optical studies. Th1s source-filter combination will limit the band width of study to the area in the light spectrum where absorption is minimum $[11,18,30]$. The incident light wavelength used in this study was in the band of minimum absorption so as to reduce the variation of absorption, and to concentrate attention on the variation of the scattering properties of sea water. 

A direct measurement of absorption is a problem because 1t involves energy transformation, and the degree of change per unit volume is less than the error in any acoeptable method of measurement.

The basic reason for the difficulty in determining the absorption coefficient accurately is that absorption is a function of wavelength, temperature, pressure, the medium, and the particle concentration. Theoretically, the absorption coefficient of distilled water under standard conditions should be constant. Unfortunately, there is wide variation in the values reported by several researchers [1]. Th1s is belleved to be due to non-standardized methods and equipment.

The total absorption coefficient, $a$, can be obtained by using Equation (2); the attenuation coefficient, $\alpha$, is obtained with Equation (1), since the measurement of attenuation is not difficult, basically. Several instruments have been devised for this purpose [22]. The scattering coefficient, $S$, is determined through separate measurement.

\subsubsection{Light Scattering}

As light energy passes through a medium, it encounters

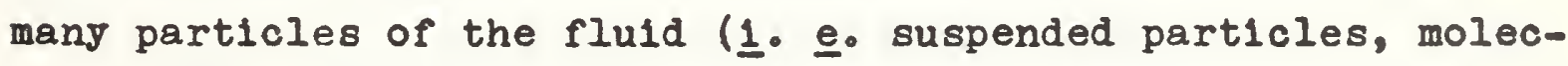
ular structure of the medium, etc.). According to Haltiner and Martin, some of the particles (dipoles) may have centers of positive and negative electric charge displaced one from another [10]. The dipole will vibrate sympathetically at the frequency of the incident electromagnetic energy. The result is that the particle radiates light energy in all directions. 

Attenuation is a function of the wavelength of the inc1dent light energy. Duntley and Koslyaninov show, for a wavelength of 480 millimicrons (1․ㅡ. Visible blue light) with a collimated incident beam, approximately 60 percent of the attenuation can be attributed to scattering and 40 percent to absorption $[24,27]$. This choice of monochromatic wavelength is discussed by Tyler, et. al., as being in the band of minimum absorption by distilled water $[24,25]$. This band is bounded by 480 millimicrons for distilled water and approximately 590 millimicrons for coastal sea water. In addition to its variation with wavelength, light attenuation also varies with scattering and absorption as already discussed. The authors have used monochromatic or limited wavelength band incident light so as to minimize the variation of attenuation.

Absorption by the particulate material in sea water is small, and may be 1gnored according to Tyler [11, 24]. The variation of absorption with temperature and pressure can be eliminated by using constant temperature at one atmosphere, in a laboratory-controlled experiment, and comparing these results with in situ measurements. This procedure reduces the varlation of the scattering phenomenon.

Some scattering (see Figure 1) is the result of the incident energy encountering the medium itself. It can be considered molecular scattering and is described by Rayleigh theory. Duntley indloates that molecular scattering is of equal magnitude in the forward and backward directions for a collimated light source in sea water [27]. He also states that molecular scattering accounts for about seven percent of the total 

observed scattering. This means that the molecular scattering is generally'several orders of magnitude smaller than particle scattering, in the forward direction.

The establishment of a specific total volume scattering coefficient is also a complex problem, but it can be measured directly. Consideration must be given to the incident energy wavelength as well as to the particle size, particle concentration, and energy dispersion. Total scattering is defined by the following functions:

$$
\begin{aligned}
\sigma(\theta) & =J(\theta) / H V(\theta) ; \\
S & =2 \pi \int_{0}^{\pi} \sigma(\theta) \sin \theta d \theta ;
\end{aligned}
$$

$\theta$ is the angle at which the intensity is measured relative to the source axis, $J(\theta)$ is the intensity measured at the angle $\theta, H$ is the input intensity per unit area, and $V(\theta)$ is the unit volume determined by the beam volume and the subtended recelver surface area. The units of $\sigma(\Theta)$ are length ${ }^{-1}$ (see Pigure 2).

1.2.4 The Effect of Particle Size, Particle Concentration and Wavelength on scattering

The Mie theory of scattering states that scattering is a function of particle size, $r$, and incident energy wavelength, $\lambda[19]$. These two parameters may be related in terms of a parameter, B, as in Equation (5):

$$
B=\frac{2 \pi r}{\lambda}
$$



When scattering particles are large in comparison to wavelength, $(B \geq 8)$, the scattered intensity is a complex function of the incident energy wavelength, relative index of refraction of the scatterers, and the angle of measurement. The relation. ship of these factors is described by the Mie theory, and varies as $\left(\lambda^{-\gamma}\right)$. The exponent $\gamma$ takes on values which range from zero for distilled water to four or five for very turbid water [19]. Values of the theoretical function $B$ are avallable, tabulated by the Bureau of Standards et. al. $[2,3,12]$. For particles comparable in size to the wavelength, the scattering function decreases in complexity. and is described in tables of the functions as indicated above. When the scattering particles are much smaller than the incident ilght wavelength $(B<<1.0)$, the relationship reduces to the simpler Rayleigh theory. In all of the scattering theory for $B>1$, the use of polarized source light reduces the complexity of function computation.

When $B$ has a large value, the resulting scattering curves show a preponderance of forward scattering, as noted in seco tion 1.1. As B decreases, the scattering pattern becomes more nearly symmetrical about the intercepted volume, and is symmetrical for molecular (Rayleighen) scattering. When considering the light intensity change in a scattering medium due to scattering only. the turbidity or fractional decrease in intensity is written as

$$
N_{r} / N_{0}=e^{-k \pi r^{2} n l}
$$



In this relationship, $n$ is the number of particles per unit volume; $K$ is a function of $B$ and the relative index of refraction, $m ; r$ is the particle radius; and $l$ is the path length traveled by the transmitted energy. Thus, for very small (molecular) particles:

$$
\mathrm{Nr} / \mathrm{N}_{0}=\frac{16 \pi^{4} r^{6}\left(m^{2}-1\right)}{\lambda^{4} l\left(m^{2}+2\right)}\left(1+\cos ^{2} \theta\right)
$$

Integration over all values of $\theta$ and substitution into Equation (6) results in the following expression:

$$
\mathrm{Nr} / \mathrm{N}_{0}=e^{\left[\frac{-128 \pi^{6} r^{5}}{3 \lambda^{4}}\left(\frac{m^{2}-1}{m^{2}+2}\right)^{2}\right] n l}
$$

The variation of $K$ for values of $M$ and $B$ is shown in Figure 3.

If light transmission were to be measured through a system of randomly dispersed particles, it would be best to select a wavelength where absorption is minimum and lessen the complications for evaluation of light scattering. If, on measuring the decrease in intensity. the intensity is found to vary as $\gamma^{-4}$, minimum absorption could be assumed. However, the generally accepted method for computing absorption is by calculating the difference between total attenuation and total scattering.

There is no complete theory at this time to treat large, irregularly shaped particles; however, most shapes can be reasonably approximated by spheres if the particles are not too large.

1.3 Station Oceanographic Climatology The authors feel that an oceanographic olimatological 

survey is required to describe the sampling area and to be certain that measured values of temperature and salinity are va11d.

We have prepared a brief description of the sampling area. The data used were those for which turbidity measurements were avallable. The description presented is from data obtained from oceanographic field sheets covering a four-year period (1951-1955) at Hopkins Marine Station of Stanford University in Pacific Grove, California. The data were processed and analyzed with the BusBdis statistical accumulation and correlation program on the USNPGS CDC-1604 computer.

Temperature and salinity were analyzed as to depth and month. The results are presented in Table 1 for the months during which the field sampling was performed. The extinction coefficient data given (Figure 4) are for the entire clima. tological sample rather than the months of the field work.

Figure 4 shows the temperature and salinity profiles for December, January, February and March, and the same data median values are shown in Table 1.

January shows the weakest vertical temperature gradient as a result of the more intense winter stirring and the warm northward flowing Davidson Current. Not shown in Flgure 4 , but in the analysis of the entire five-year sample, is a vertical temperature gradient of two degrees celsius in the fifty-meter surface layer.

A well-mixed surface layer is shown for December and January only, and a weak salinity gradient is evident for this 

period as well.

An investigation of light attenuation versus cloud cover was conducted; first, to determine the validity of the observations; and second, to determine the shape of the extinction curve in the vertical at the chosen location. 107 observations made with an hydrophotometer were avallable. Two values were available for each observation; one for incident sunlight at the sea surface, and one for l1ght existing at each depth.

A small negative correlation $(0.16)$ was found between the surface light and oloud cover. Whether this correlation should be this small or if it should be larger is difficult to say, as there is certainly some light even with overcast skies. There is also the possibility of large observational error here, because of variability in observational technique. The next step in the light investigation was an examInation of light versus depth. This gave a negative correlation of 0.69 . Figure 5 is a graph of relative radiance (relative to incident insolation at the surface) by depth, using the 155 available observations. Flgure 5 shows that the relative radiance"approaches zero as depth increases, and allows some quantitative oomparison. Curve 1 of Figure 518 a plot of percent of incident sunlight versus depth, measured by Tyler in 1957 with a photometer oriented at the zenith angle in fresh water, and the sun at $65^{\circ}$ altitude [11]. Curve 2 is a similar plot for the data of the present study, which has hand-fitted to extinction hydrophotometer data. The observations were made at 10:30 a.m. \pm 30 minutes, so 1t is safe to assume that the sun alt1tude was not much different 

from $65^{\circ}$. Aside from the variation due to sun altitude, there are unknown instrument differences, and the data available were not of the precise nature of Tyler's data. These factors, along with the difference in water type, contribute to the difference of the slope of curves 1 and 2. Curve 3 is hand-fitted to the station climatological turbid1ty data. It is observed from the curves of Figure 5 that Monterey Bay water is relatively turbid.

Here, we can point out that a very shallow thermocline such as exists in the Bay water would hold the more turbid water close to the surface, and tend to change the radiance curve in the manner noted in Figure 5 for this study. This turbidity appears to be concentrated in the upper 25 meters, as there is practically no radiance below 25 meters as indicated by curve 3.

Another contributing factor in this regard is the difo ference in incident sunlight. In the present investigation, the mean incident cloud cover for the 155 observations is 53 percent, while Tyler's curve was established in a single clear day.

The resuiting conclusions as to radiance of Bay water is that in the vertical, total extinction is similar in many respects to $\mathrm{Tyler}^{8} \mathrm{~s}$ data ${ }_{9}$ but that the water is relatively more turbid in the upper 25 meters [11]. 

2. Equipment Description

2.1 Sea Water Attenuation Hydrophotometer (Alpha Meter)

This instrument was supplied by the V1sibility Laboratory of Scripps Institute of Oceanography, San Diego, Cal1fornia. It is designed to measure total horizontal light attenuation. It consists of a constant light source with a monitor cell in a sealed container on one end of a three inch I Beam, as shown in Figure 6. On the other end of the bar is a photocell, also enclosed in a watertight container, with a Wratten Filter number 57. The electric power $1 \mathrm{~s}$ supplied on board the USNPGS Hydrographic Research Vessel by two 500-k1lowatt generators. The control box contains two potentiometers to display transmitted and received intensity. The Alpha Meter is first standardized in air to calibrate the two meters with the power supply. Then the apparatus is lowered just below the sea surface and is standardized at this position and the first reading is made. Since this meter determines horizontal light attenuation, attention must be given to maintaining a horizontal position during all readings. Lowering the Alpha Meter to specific meter wheel depth readings allows data to be taken at depths down to a maximum cable length of 50 meters.

The Wratten Filter number 57 passes maximum intensity at 0.5364 microns with a half width of 0.050 microns. This band is in the region of maximum transmittance of light in sea water. Forward angle scattering was measured using light in the same wavelength band. 

2.2 Insolation Extinetion Hydrophotometer, G. M。 Mfg. \&

Instrument Corp. Submarine Photometer Model $15 \mathrm{MO} 4$

This instrument was used to determine solar radiation extinction with depth, which gives a measure of upper layer turbidity. The basic equipment, as shown in Figure 7, consists of two photocells; one, a deck monitor to measure surface incident solar radiation, the second to measure solar radiation at some selected depth. The information is displayed by means of two potentiometers mounted in the deck control box. This allows the operator to obtain significant data points at pre-selected depths, and to determine the depth at which solar radiation is totally attenuated. A Wratten Filter number 57A is used with the in situ photocell, which has a peak transo mittance at a warelength of $0.5340 \mathrm{microns}$ and a half width of 0.060 microns, which overlaps the band width of the Alpha Meter Wratten F1lter number 57.

With the Insolation Extinction Hydrophotometer and the Sea Water Attenuation Hydrophotometer, it is possible to obm tain vertical and horizontal attenuation in the upper layers simultaneously. There should be an extinction maximum in the areas of high concentration of particulate matter. It is also possible to compare these data with other oceanlc areas, as w111 be seen later.

\subsection{Scattering Analysis Apparatus}

2.3.1 Constructed Laboratory Model

As shown in Figure 8 , there are four major components to this variable angle scattering analysis apparatus: a Mero cury Arc lamp; and Eldorado Differential Photometer, Model 210, 

with two photocells, (capable of recording as small as two micro-micro lumens); a Leedsworthrup Analog Chart Recorder; and the varlable angle protractor scattering table with one liter flask, aperture, wavelength discrimination filter, polarization neutral screen, and focusing (collimation) lens. The Mercury Arc lamp produces a narrow beam which contains monochromatic Iines at $0.6907,0.6234,0.5791,0.576959,0.5461$, $0.4960,0.4916,0.435835$, and 0.404656 microns. The lines at $0.576959,0.435835$, and 0.404656 microns are the only lines whose intensity are of practical use. Using a Wratten Filter number 57, it is possible to eliminate all the lines except the desired wavelength of 0.576959 microns.

It is desirable to collimate the beam to eliminate any beam divergence over the measuring path. This can be done by using a $3 / 32$ inch aperture with a rive-inch focal lens. Proper alignment and positioning of aperture and lens gives nearly zero divergence over the path used (beam width is $0^{\circ}-8^{\prime}$ at the receiver). A pair of adjustable polarizing lenses are positioned in the beam to obtain control of the absolute intensity which is received by the Eldorado Differential Photometer photocells. The flask is positioned over the center of the protractor scattering table with 1 ts relative position always checked for consistancy to eliminate any changes in beam path due to glass-water interface refraction.

One of the photocells is positioned on the beam path. The second is secured to the rotating arm of the protractor scattering table. An electric slow speed AC motor is geared to the rotating arm so as to provide the necessary constant 

rotation of the variable position photocell. The arm movement is slow enough to present a useful curve on the LeedsNorthrup Analog Recorder, giving readings on a continuous curve rather than at discrete points.

To align the apparatus, it is necessary to diminish the signal received by the fixed arm photocell to a value which allows adequate scale freedom on the Eldorado Differential Photometer. Allgnment is then made with the air-fllled flask to eliminate flask refraction effect. This is repeated with distilled water, so that the ultimate data will be relative to the distilled water.

As is seen in Figure 9, this apparatus can process a large number of one 11 ter samples in complete darkness, with a permanent record of the results. The product is a continuous curve of scattering at angles varying from that perpendicular to the beam axis to along the axis. 



\subsubsection{Aminco Light Scattering Microphotometer}

The Aminco Microphotometer, of the American Instrument Company, Inc, Silver Spring, Maryland, was used for samples of less than one-liter volume and for individual point studies (1. $\underline{\text { e. at }}$ particular angles). The apparatus, shown in Figure 10, consists of the same general components on the larger scattering apparatus with the exception of not having a differential photometric capability. The same type AH4 mercury arc lamp is the light source with a Wratten 57 Filter and a three-inch focal length collimating lens. The recelver photocell which sits on a variable-angle plate receives and displays the intensity of light scattered from the sample at various angles with respect to the light beam. The apparatus gives good small-angle forward scattering resolution for a 62.8 cubic-centimeter sample, but because of the necesstty to pre-position definite angles manually, and the sample size limitation, it was used as an auxiliary and checking system.

The procedure is to determine the light beam intensity first in air, and then with a sample of distilled water at each degree relative to the beam axis. When this calibration is complete, any sample can be investigated at any angle desired. After completion of sample analysis, another distilled water sample is analyzed for beam attenuation on the axis to evaluate the meter drift.

\subsection{Salinity Determination Apparatus}

The Hytech Model 621 Inductive Salinity and Conductivity Meter was used to measure salinity. The Meter uses 

the magnetic induction method to compare the conductivity of a sample with that of Copenhagen water. This apparatus compensates automatically for temperature differences bew tween the sample and the standard; if the differences are small, allowing all reagents to come to room temperature will satisfy the temperature requirements. The salinity range capability of the Meter is from 0 to $44 \%$ with an accuracy of 0.003 . percent salinity, which includes errors which may be made during normal handing procedures using a 50 cubic centimeter sample.

2.5 Phosphate Determination Apparatus

The phosphate determination was conducted using a color comparison technique with the Beckman DU Spectrophotometer. The spectrophotometer compares a sample, after the addition of an acidic solution of ammonium molybdinate, plus an agent such as stannous chloride to reduce the complex phosphom molybidic acid to a blue colored substance, with a prepared set of samples of known concentration. This colorimetric quantitative analysis determines differences in the colors which are proportional to the concentration of the phosphate in the sea water sample. The accuracy of the results is about 5 percent throughout the phosphate concentration range, which generally lies between 0.00 and a maximum of 3.00 millic grams per liter for sea water. 

3. Procedure

3.1 Sample Collection and Attenuation Measurement

The primary purpose of this investigation is to discover the nature of the vertical variation of light scatterers in Monterey Bay. Previous work in this field has pointed toward a need to analyze forward-angle scattering, and the techniques and apparatus were designed with this in mind.

Working on a foundation of information published by Burt, Tyler, et. al.o the authors concluded that uncontaminated water samples collected in the field would retain their optical properties if analyzed quickly enough to preclude decomposition of suspended organic matter, and they could be collected by Nansen bottle cast $[9,17,22,23]$. Spilhaus showed, in his study of the areal distribution of scattering, that it was practicable to use a shipboard laboratory device to measure scattering in samples collected by Nansen bottle cast [35].

Sample depth was determined by a combination of wireangle computation and bathythermograph slide interpretation.

The water samples were collected on 21 December, 1964: 6 and 8 January, 26 January, and 19 February, 1965. The first three days were during a stormy weather period and the surface waters were well-mixed. The last two days were during a relatively calm period and the surface water mixing was less intense. The spread of collection days was due to severe weather conditions.

A station was selected offshore on the rim of the Monterey Submarine Canyon in about 900 fathoms of water. This station was chosen because of its location at the Bay entrance, 

with expected low terrestrial polution. Also, previous sunlight extinction data were avallable for comparison, and the oceanographic climatology of the area had been established (note section 1.3). Th1s station 1 s located at $36042^{\prime} \mathrm{N}$ and $122^{\circ} 02^{\circ} \mathrm{W}$, and all samples were taken within two miles of this location, with positioning by visual bearings (see Figure 11).

Three types of light transmission measurements were made:

(a) horizontal attenuation of a light beam, in situ, using the Alpha Meter (Figure 6).

(b) vertical extinction of sunlight, using the hydrophotometer in situ (Figure 7).

(c) horizontal scattering of a collimated light beam, with scatterometers in a shore laboratory, shown in schematic form in Figure 2.

All light measurements were made at nearly the same wavelength. The procedure at the Bay station was:

1. Horizontal attenuation measurement at five-meter intervals to cable limit.

2. Sunlight extinction measurement at five-meter intervals.

3. Nansen cast at standard depths.

4. Bathythermograph cast.

Immediately upon returning to port, the water samples were taken to a USNPGS laboratory for scatterometer measurement. Later, the salinity and phosphate determinations were made, with careful handling of samples and storage at a constant $66^{\circ} \mathrm{F}$ temperature. 

3.2 Forward Angle Scattering Measurement

All scatterometer readings were preceded by readings with the chamber first alr-filled for meter calibration and then fllled with distilled water to provide relative values for Mie scattering determination. Individual readings for alr, dist1lled water, or sea water, consist of a base transmission reading made with a cell on the beam axis to check alignment and intensity and a curve of scatterometer readings from angles of $90^{\circ}$ to $180^{\circ}$ relative to the beam axis.

To establish the vertical variation of suspended material, as many variables as possible must be eliminated. This may be accomplished by:

1. Selection of a narrow wavelength band (approx1mately monochromatic) to assure minimiz absorption (by wavelength selection) and eliminate variability of Raylelgh scattering. The most effective wavelength has been found to be -56 microns, for bay water. A comparable wavelength value for turbid coastal water is close to 0.58 microns, and for distilled water, about 0.48 microns. Natural sea water acts as a monochromator in this region, with minimum absorption generally near a wavelength of 0.53 0.54 microns. Avallable data for comparison of results and standardization can be used if a monochromatic light source is used $[11,24,35]$.

2. Collimation of the light source to assure predominance of forward scattering and placing 

the scattering dependence on scatterer size and concentration.

In the scattering apparatus, $\sigma(\theta)$ was measured at angles and plotted as relative signal intensity versus angle. The position of the curve for each sample should provide a large particle scatter coefficlent. Comparison of slopes of the curves should give an indication of particle size distribution. This assumes the Baylelgh scattering is constant from sample to sample, and is the same for either distilled or sea water.

In line with Tyler's paper on angular resolution in scattering measurement, a beam of high collimation with vertical recelver slits was used in scattering measurements [26]. 

4. Data

4.1 Light Attenuation Data

Data shown in Table 2 were taken by means of the Scripps Alpha Meter, as outlined in sections 2.1 and 3.1 , and were observed in situ. The difficulties encountered were due to the large wire angle caused by existing weather conditions. This caused the cable welght to be exerted on the pressure connectors of the cables. This additional welght, plus the boat motion, brought about occasional cable separation. On the 8 th of January, the wire angle was zero and the cable parted at the connectors due to cable weight and handilng. On the 26th of Jano uary, the cable developed an internal break at a splice point, making data-taking impossible. On the 19th of February the apparatus operated perfectly.

4.2 Light Extinction Data

Data show in Table 3 were obtained in situ, using the USNPGS Solar Radiation Extinction Hydrophotometer, described In sections 2.2 and 3.1 . The first two crulses were late in the day and the weather was bad, with high winds and heavy cloud cover, and this gear was not used. The next two cruises had good weather conditions and the resulting data were taken at mid-day on both days, at about 1415 local time. On the 19th of February the apparatus was lowered just as the boat drifted into a fog bank which decreased the amount of incident radiation. On this day the water was extremely turbid. 

4.3 Scattering Coefficient, Density, Phosphate, Particle Concentration Data

Data shown in Table 4 were taken in a USNPGS laboratory with equipment and procedures explained in sections $2.3,2.4$, 2.5, and 3.2. The scattering data were first taken on a table arrangement shown in Figure 8. The procedure was awkward because the measurements had to be made in the dark. A constant speed drive was necessary to glve the required constant angular velocity for the scattering measurements. Difficulties arose because the scattering intensity varied through three orders of magnitude, requiring the shifting of meter scales at the correct moment, in total darkness. If time were available to correct the design of this scattering apparatus, the present undesirable features could be eliminated. It might prove to be a most accurate scattering measuring device because of the high sensitivity of the differential photometer.

The samples obtained on the 26 th of January and the 19th of February were analyzed, using the Aminco scattering meter described in Section 2.5. The Aminco Meter is less sensitive than the constructed protractor table. This drawback is more than compensated for by the use of small samples, working in a lighted room, and measurement at discrete points rather than continuously.

A plot of the scattering function $\sigma(\theta)$ versus angle has a distinctive shape, (see Figures 12, 13, and 14). The shape of this curve is controlled by the particle size, and the area under the curve is proportional to the concentration of the scatterers. The National Bureau of standards, et. al. have 

published tables of the scattering function $\sigma(\theta)$ and a reo lated value $K_{0}\left(K=S / B^{2}\right)$ for many combinations of the paro ameters of particle size $\left(B=\frac{2 \pi r}{\lambda}\right)$ and relative refractive index $m[2,3,12]$.

The tables of $K(m, B)$ are based on concentrations of one particle per unit volume of liquid medium. The $\sigma(\theta)$ values mentioned above were computed by evaluation of the Mie scattering equations. The curves showing the theoretical values are shown in Figures 12, 13 and 14. The Mie curves for the Nansen bottie samples (shown in Figure 15) are similar to the theoretical curves mentioned above, in shape.

A segment-by-segment integration of the measured scattering using Equation (4), shows that approximately 75 pero cent of the volume scattering coefficient is achiered in the angles from the beam axis between $160^{\circ}$ and $180^{\circ}$ [35]. This is also the region where the large particle scattering effect is greatest (see section 1.2.3). Using large plastic overlays of Figures 12, 13, and 14, 1t was possible to match the measured curve slopes from $179^{\circ}$ to $160^{\circ}$ to the theoretical single-particle curves. This provided an estimate of particle size and relative refractive index for each sample curve. 



\section{Data Interpretation}

The values of scattering intensity and volume scattering coefficlent are show in Table 4. Using these data, the vertical varlation of the volume scattering coefficient, the relationship between the scattering coefficient and sea water density and inorganic phosphate, and the approximate size and concentration of suspended particulate matter can be determined。

There is a rellance on certain established facts and assumptions. Two fundamental 1deas are: 1. absorption in sea water is minimum for collimated light; 2 . the best wavelength band for coastal water transmission is .48 to .59 microns, (see section 1.2). The wavelength used in this study is . 5364 microns。

The assumptions which were used are:

1. Scattering by particles small compared to the wavelength is negligible.

2. Scattering is constant for distilled water at a constant temperature, for a particular water sample.

3. The large particles are assumed to be of homogeneous composition and similar to one another in chemical and physical makeup, and consequent$1 y$, have the same relative refractive index.

4. Absorption by suspended matter is small relative to scattering.

5. Particles in each sample are assumed to be uniformly sized and uniformly dispersed. 

6. Scatterers will remain in suspension and unchanged for a short time (about four hours) after sample collection.

It is believed that most of the material found in sea water of the particular size under investigation has a relative refractive index in the range 1.2 to 2.0 , particularly the organic material.

On the last sampling day, the bottom two bottles picked up samples from a turbidity current along the canyon wall. Upon retrleval, the bottom weight was dragged along the canyon wall and a small sample of mud and pebbles was obtained. It was felt that a sediment analysis of this sample would give an indication of the nature of the suspended material in the sea water above. The analysis of the bottom sample by Ii eutenant Gordon Monteath showed over two-thirds of the sample to be in the size range of less than 12.5 microns (pero cent by welght) [36]. The material was a greenish-brown, very fine silty sand matrix, surrounding well-rounded pebbles of granodiorite and quartzite. Over 60 percent of the sample was quartz and feldspar (75 percent of this was light pink in color and in the size range less than 12.5 microns). There were notable amounts of mafic minerals, shell fragments, blotite, and aggregates-coprolites.

The majority of these minerals and other substances have an index of refraction which is in the range 1.20 to 1.65 . Therefore, the use of $m$ values between 1.20 and 1.44 seems to be appropriate in this study。 Referring to the $K$-field chart, Figure 3, the relationship can be seen between $K(m, B)$, 

(which is proportional to volume scattering), particle size, $r$, and the index of refraction, $m$. This chart shows that the larger particles (which most affect the scattering) have an $m$ range between 1.20 and 1.44 . The region of maximum $K$ value (for $B<12$ ) is found in a zone of decreasing $B$ values and increasing $m$ values. As the index of refraction increases, the greatest scattering (greatest $K$ ) is found to be associated with particles of smaller and smaller radius. After the selection of the appropriate $B$ and $m$ values for each sample curve, a plot of the theoretical K-field was entered and the applicable $K$ value was determined. Using Equations ( 1 ) and (2) and assuming absorption to be negligible gives

$$
N r / N_{0}=e^{-5 l}
$$

Setting Equation (9) equal to Equation (6) gives

$$
s=k \pi r^{2} n
$$

The measurement of scattering intensity, $J(\theta)$, and solution of Equation (3) and Equation (4) gave us a measured value of $S$. We have a fitted $K$ and an estimated $r$ value (determined from $B=\frac{2 \pi r}{\lambda}$ Section 1.2). Solution of Equation (10) for each sample gives a measure of particle concentration n. These are shown in the data compllation, Table 4.

Concentration is seen to be proportional to scattering and inversely proportional to particle size. Values shown in Table 4 for the two parameters, radius and scattering coefficient, were obtained by different means. 

Figure 21 shows a scatter diagram of $S$ versus $h$, according to particle radius. There appears to be a separation in the relations for particle size of one to two microns and those greater than two microns. Except for very small partio cles, scattering increases with particle radius for a given concentration. This relationship is more pronounced for the one to two micron size than for particles larger than two microns. It is also shown in the Kofleld (Figure 3) that the $K$ value increases for increasing particle radius; in the size range of interest (greater than one micron).

An examination of the particle size variation shows no clear relationshlp with depth, considering all samples. Particle sizes obtained from curve fitting are mainly in the size range between 1.6 and 2.4 microns. The reader will note from Figures 16 to 20 that a definite difference in vertical profiles of salinity. phosphate, and sigmat exists for the last two sample days as compared to the first three days. Weather conditions would tend to cause the upper water layers to be well-mixed on 21 December, and 6 and 8 January, while layered conditions existed on 26 January and 19 Febo ruary. One would expect, then, a uniform size distribution in the upper layers for the first three sample days. Looking at particle size from the standpoint of a layered system, it was found that on the three sample days with mixing, the upper 50 meters (considered as a mixed layer) shows a more uniform particle size than is shown on the last two days. This sugo gests that the resulting variation in scattering in the upper layer may be due to varlations in particle concentration. 

Flgure 22 shows the scattering, field for values of particle radius and particle concentration. This emplrical relationship should be verified by future experimentation.

It is difficult to say how the surface area-to-mass ratio of particles will be distributed with depth. The relative refractive index of lightwelght particles with the nature of wax or animal fat would be about 1.2. The same index for mineral material such as quartz might range up to 1.5. The small index difference is considered in the curve fitting. The surface area-to-mass ratio should have wide divergence for organic and mineral particles. The computed particle sizes would lead us to beliere that we should be able to detect an increase of size with depth under calm conditions. It is possible that the large particles we see are of very light material and not greatly affected by gravity. The majority of the scattering particles however, probably are not living zoo or phyto-plankton. A previous study by the authors of the most common Monterey Bay planktonic forms shows a range in size upward from 15 microns.

It was hoped that some clear relationship between scattering and phosphate concentration might be found; (see F1gure 23). It is noted that there is little variation of scattering with phosphate amount for the two sample days with layering in the upper 50 meter layer (Table 4). More variation is seen below 50 meters. For the three days with mixed waters there can be seen a negative correlation between scattering and phosphate in all samples, with a tendency for greater scattering in the upper 50 meters. There is no in- 

dependent verification of the latter relationship; however, some support is provided through data from the first two days (the well-mixed condition) when phosphate was observed to be high in the surface waters.

No definite conclusion can be formed concerning the relationship between particle concentration and depth. There appears to be a tendency for greater concentration of particles above 50 meters for the two days exhibiting an unmixed layer. The computed scattering coefficlent is relatively large at the base of this unmixed layer.

Little useful information on scattering can be obtained from the attenuation and extinction data because of poor data overlap with the scattoring analyais. A check of the data shows the resulting absorption to be reasonable when checked using the attenuationoscattering difference. 



\section{Conclusions}

The clear relationship expected between scattering coefficients and density and phosphates did not develop from this study, although a tendency is suggested in this direction. Perhaps a future study usting a much larger sample size will verify the trend. There seems to be a light scattering layer (comparable to the deep scattering layer for sound transmission) at about 50 meters. This layer appears to be partially destroyed by mixing in the upper layers from storm activity. This light scattering layer appears to be associated with the upper margin of a pynocline which would buoy small particles and organisms and tend to be a collection area for these less dense materials. Seasonal variation of this pynocline will definitely affect the optical water mass characteristic. This in turn will affect the variation of solar radiation present in the region of the pynocline. This scattering layer will also affect the variation of attenuation with depth due to increased scattering and absorption. It is possible that this layer has a high concentration of planktonic organisms which exist in the region of less than one percent incident solar radiation.

From the data (Table 4) on particle size and concentration, we conclude that the methods used are sound and the results valld. The techniques could be adapted to shlp-board use for the rapid processing of a large number of samples. The final computations are, however, tedious and time-consuming and computerization of all computations after initial intensity readings is desirable. 

To provide more meaningful results from future studies of this type, the authors feel that attenuation hydrophotow meter measurements are necessary for all levels where samples are taken. This provides a check on the order of magnitude of scatter coefficients and any layering discovered can be closely sampled to obtain data on parameters affecting the variation of scattering. If a verification is achieved, Figure 23 would be useful for estimates of particle radius and particle concentration if a scattering coefficient is ava1lable.

In future studies the authors recommend:

1. The use of a coherent light source with variable wavelength selection.

2. The use of a monochromatic collimated light source with at least two discrete wavelength selections to give beam definition and the ability to determine particle size and concentration without the undesirable and subo jective curve fitting technique.

3. A chemical and microscopic analysis be made with in vitro samples to be correlated with associated scattering data.

The authors wish to express their profound gratitude to Dr. Glenn H. Jung for his assistance. Wl thout his staunch support, a problem of this magnitude could not have been completed. The technical assistance of Dro Gerald D. Ewing in the field of light transmission in sea water is greatly appreclated. The work of Dr。 Raymond I. Kelly and Professor 

Sidney H. Kalmbach in optical physics; Dr. Charles F. Rowell in chemical oceanography; Richard Wo Haupt, Commander, United States Navy, in oceanographic instrumentation; and Assistant Professor Warren Denner all contributed to this complex study. We wish to express our sincere thanks to Gordon Monteath, Lieutenant, United States Navy, for his sediment analysis and his assistance in sampling on 21 December, 1964, and 6 January, 1965. The weather conditions on both of these days were extreme, and without his help, the operation might have met with instrument loss before the sampling and experimentation got started. The authors wish to express their appreciation to Mr. Roswell Austin of the Visibility Laboratory at Scripps Institute of Oceanography for the use of the Alpha Meter. For the computer techniques and programing advice, we are deeply indebted to Mrs. William L. Johnson. Credit should be given to our wives, Mrs. Charles Bassett and Mrs. Harry Furminger. Their typing, editing, data breakdom and moral support was outstanding and essential. 



\section{BIBLIOGRAPHY}

1. Sverdrup, Ho U. Johnson, M. Wo, Fleming, R. H. The Oceans. Prentice Hall, Inc。, 1942.

2. National Bureau of Standards. Tables of Scattering Functions for Spherical Particles. United States Govern. ment Printing Office. January, 1949.

3. Grumprecht, R。 O。, Sung, No Io, Chin, Jo No, SIlepevich, C. M. Angular Distribution of Intensity of Light Scattered by Iarge Droplets of Water. Journal of the Optical Society of America, $v$ 。 42, November, 19518 226-231.

4. Johns Hopkins University. The DualoFilter Hyarophotometer, by J. Williams. March, 1953. Technical report number 5 .

5. Davis, C. No Survey of Scattering and Iight by Particles. British Journal of Applied Physies, v。 5, Sup. 3, 1954.

6. Lewis, P. Co, and Go Fo Lothian. Photoextinction Measurements on Spherical Particles. British Journal of Applied Physics,, 5 , Sup。 3, 1954.

7. Johns Hopkins University. The Trimilter Hydrophotometer, by J. W1Iliams. June, 1955. Technical report number 9.

8. Barum, E。 Go The Ecology of Sonic scatterers in the Monterey Bay Area, California. Dissertation, Stanford Universlty, November, 1956.

9. Burt, $W_{0} V_{0}$ On Attenuation of Light in the Sea. Journal of the Marine Blologs Association of the United Kingdom, v. 36; 1957 。

10. Haltiner, $G_{0} J_{0}$ and $F_{0} I_{0}$ Martin. Dynamic and Physical Meteorology. MeGrawoHili, Inc. 19578790104.

11. Tyler, Jo E. Monochromatic Measurement of the Volume Scattering of Natural Waters. Journal of the Optical Soclety of America, vo 47. August, 1957: 745074?.

12. Ashley, L。 $E_{0}$ and $C_{0} M_{0}$ Cobbo single Particle scattering Functions for Latex Spheres in Water. Journal of the Optical society of America, $v$. 48, Apri1, $19588261-268$.

13. Clark, Go I. and R. Ho Backus, Measurement of Light Penetration in Relation to Vertical Migration and Records of Luminescence of Deep Sea Animals. Deep Sea Research, v. 4, 1958: 1-14. 

14. Woods Hole Oceanographic Institution. Measurement of the Spectral Distribution of Iight Underwater, by C. J. Hubbard。January, 1958. Report no. 58-6.

15. Rakestraw, N. Wo Particulate Matter in the Oxygen Minimum Layer. Journal of Marine Research, v. 17, 1958: 429-431.

16. Woods Hole Oceanographic Institution. Optical studies or Particulate Matter in the Sea, by $D_{0} H_{0}$. Shontig and B. H. Ketchum. February, 1958. Report no. 58-15.

17. University of Washington, Department of Oceanography. Specific Scattering by Uniform Minerogenic Suspensions, by W. V. Burt. January, 1959. Report no. 42.

18. Tyler, J. E. Natural Water as a Monochromator. Limnology and Oceanography, V. 4, January, 1959: 102-105.

19. Orr, Co, and J. Mo Dallavalle. Particle Size Measurement from Radiation Transmission: Fine Particle Measurement. Mac-Millan, 1959.

20. Laevastu, To, Factors Affecting the Temperature of the Surface Layer of the Sea. Sociates Scientiarum Fennica, Helsinki, 1960 .

21. U. S. Naval Research Laboratory. A High Resolution Investigation of the Relative Spectral Attenuation coefficients of Water, by $I_{0} F_{0}$ Drummeter and Go L. Knestrick. May, 1961. Preliminary report no. 5642.

22. Tyler, Jo E。 and RoWo Preisendorfer. Transmission of Energy Within the Sea. The Sea, V. I, Interscience, 1961.

23. Tyler, J. E. On the Measurement of the Scattering Function of the Sea. International Union of Geodesy and Geom physics Symposium on Radiant Energy in the Sea, Helsinki. Monograph no. 10, June, 1961: 40-45.

24. Tyler, Jo E. Scattering Properties of Distilled and Natural Water. Ilmnology and Oceanography, V. 6, October, 1961: $451-456$.

25. Tyler, Jo E. Measurement of Scattering Properties of Hydrosols. Journal of the Optical Society of America, v. 51, November, 1961: 1289-1293.

26. Tyler, Jo E。 and Co Howerton, Instrument for Measuring the Forward Scattering Coefficient of Sea Water. Limnology and Oceanography, V. 7, July, 1962: 393-395. 



\section{BIBLIOGRAPHY}

27. Duntley, S. Go Light in the Sea. Journal of the Optical Society of America, $\nabla .53$, February, 1963: $214-233$.

28. U. S. Naval Research Laboratory. Transmission of Ruby Laser Light Through Water, by J. A. Curcio and G. L. Knestrick. June, 1963. Report no. 5941.

29. Jerlov, No Go Opt1cal Oceanography. Oceanographlc Marine Blology Annual Review, 1963: 890114.

30. Hulbert, E. O. Optics of Distilled and Natural Water. Journal of the Optical Society of America, vo 35, November, 1963: 698-705.

31. Irani, R. R。 and F。 C. Clayton。 Light Scattering as a Measure of Particle Size: Particle Size Measurement and Interpretation. Wlley, 1963.

32. Hopkins Marine Station, Stanford University. Pacific Grove, California. Studies of the Marine climate and Phytoplankton of the Central Coastal Area of California, by R. L. Bolin and D. P. Abbot. July, 1964.

33. U. S. Naval Electronics Laboratory. Transparency of Coastal Waters, by R. Fo DIII and A. Gargola. 1964. Report.

34. U. So Naval Research Laboratory. Opt1cal Properties of Materials, by Go I. Knestrick, A. Go Rockman, J. A. Curcio. July, 1964: 26-27. Progress report, problem no. NOI-07.

35. Sp1Ihaus, A. Fo, Ir. Observations of Light seattering in Sea Water. Dissertation, Massachusetts Institute of Technology, February, 1965.

36. Monteath, Gordon, Lt。, USN, Environmental Analysis of the Recent Marine Sediments of Southern Monterey Bay, California. Thesis, U。 S。 Naval Postgraduate School, 1965. 



\section{APPENDIX I}

TABLE 1

\section{MEDIAN TEMPERATURE AND SALINITY}

\begin{tabular}{|c|c|c|c|c|}
\hline \multirow[b]{2}{*}{ Depth } & \multicolumn{2}{|c|}{ December } & \multicolumn{2}{|c|}{ January } \\
\hline & Temp. & Salinity & Temp. & Salinity \\
\hline $\begin{array}{r}0 \\
10 \\
20 \\
30\end{array}$ & $\begin{array}{l}54.18 \\
54.18 \\
54.17 \\
54.18\end{array}$ & $\begin{array}{l}33.06 \\
33.06 \\
33.06 \\
33.06\end{array}$ & $\begin{array}{l}53.46 \\
53.46 \\
53.47 \\
53.51\end{array}$ & $\begin{array}{l}33.11 \\
33.10 \\
33.23 \\
33.23\end{array}$ \\
\hline $\begin{array}{l}40 \\
50\end{array}$ & 52.00 & 33.09 & $\begin{array}{l}\text { not a } \\
53.49\end{array}$ & $\begin{array}{r}11 a b l e= \\
33.24\end{array}$ \\
\hline
\end{tabular}

Eebruary

Mareh

Depth Tempo Salinity Temp. Salinity

$\begin{array}{lllll}0 & 52.93 & 33.16 & 51.67 & 32.88\end{array}$

$\begin{array}{lllll}10 & 52.75 & 33.24 & 51.57 & 32.95\end{array}$

$\begin{array}{lllll}20 & 52.75 & 33.24 & 51.30 & 32.95\end{array}$

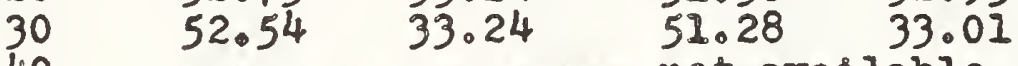

40 mot avallable-

$\begin{array}{lllll}50 & 51.51 & 33.29 & 50.02 & 33.12\end{array}$

Temperature in degrees Fahrenheit. Salinity in parts per thousand. Depth in meters. 

TABLE 2

LIGHT ATTENUATION DATA

\begin{tabular}{llllll} 
Meter & $\begin{array}{l}\text { Accepted } \\
\text { Wheel }\end{array}$ & $\begin{array}{l}\text { Iight } \\
\text { Depth }\end{array}$ & $\begin{array}{l}\text { Alpha } \\
\text { Source }\end{array}$ & $\begin{array}{l}\text { Percent } \\
\text { Reading }\end{array}$ & $\begin{array}{l}\text { Temp. } \\
\text { Reading }\end{array}$ \\
Meters & Metersity & & Arbitrary units & $e^{-\alpha} \propto$ & \\
\hline
\end{tabular}

\section{December, 1964}

$\begin{array}{rrrrrrr}0 & 1 & 17.3 & 8.5 & 0.492 & 0.71 & 54.3 \\ 5 & 4.5 & 17.3 & 8.8 & 0.508 & 0.68 & 54.2 \\ 10 & 8.1 & 17.3 & 8.6 & 0.497 & 0.70 & 54.2 \\ 15 & 11.7 & 17.3 & 9.0 & 0.520 & 0.65 & 54.1 \\ 20 & 15.9 & 17.3 & 9.2 & 0.532 & 0.63 & 54.0 \\ 25 & 20.4 & 17.3 & 9.5 & 0.549 & 0.60 & 53.6 \\ 30 & 25.1 & 17.3 & 9.8 & 0.567 & 0.57 & 53.6 \\ 35 & 29.9 & 17.3 & 9.8 & 0.567 & 0.57 & 53.6 \\ 40 & 34.8 & 17.3 & 9.8 & 0.578 & 0.55 & 53.5 \\ 45 & 39.2 & 17.3 & 10.0 & 0.567 & 0.597 & 53.5 \\ 50 & 44.8 & 17.3 & 9.8 & \text { Disconnected } & 53.5\end{array}$

6 January, 1965

$\begin{array}{rrr}0 & 1 & 15 \\ 5 & 5 & 15 \\ 10 & 9 & 15 \\ 15 & 13 & 15 \\ 20 & 17 & 15 \\ 25 & 22 & 15 \\ 30 & 27 & 15 \\ 35 & 32 & 15\end{array}$

6.0

$0.400 \quad 0.91$

54.5

8.0

0.533

0.63

52.6

158.0

0.533

0.63

52.6

8.0

0.533

0.63

52.5

157.7

0.513

0.67

8.2

0.547

0.60

52.5

158.5

0.567

0.57

Disconnected

52.6

52.6

52.5

$\frac{8 \text { January, } \frac{1965}{0}}{5}$

$\begin{array}{rrr}0 & 0 & 12 \\ 5 & 5 & 12 \\ 10 & 10 & 12 \\ 15 & 15 & 12 \\ 20 & 20 & 12 \\ 25 & 25 & 12 \\ 30 & 30 & 12 \\ 35 & 35 & 12 \\ 40 & 40 & 12 \\ 45 & 45 & 12 \\ 47 & 49 & 12 \\ 45 & 45 & 12 \\ 40 & 40 & 12 \\ 35 & 35 & 12 \\ 30 & 30 & 12\end{array}$

6.6

$0.550 \quad 0.59$

$0.617 \quad 0.48$

54.2

7.4

7.2

0.600

0.51

54.1

7.3

0.608

0.50

0.592

0.53

7.1

0.592

0.53

0.567

0.57

0.617

0.48

0.583

0.54

0.633

0.46

0.533

0.63

0.51 ?

0.66

6.2

0.500

0.69

6.5

0.542

0.61

Disconnected

54.0

54.0

54.1

54.1

54.2

54.2

54.5

54.5

54.7

54.5

54.5

54.2

54.2 

TABLE 2

\begin{tabular}{|c|c|c|c|c|c|c|}
\hline $\begin{array}{l}\text { Meter } \\
\text { Wheel } \\
\text { Reading } \\
\text { Meters } \\
\end{array}$ & $\begin{array}{l}\text { Accepted } \\
\text { Depth } \\
\text { Meters } \\
\end{array}$ & \multicolumn{2}{|c|}{$\begin{array}{ll}\text { Light } & \text { Alpha } \\
\text { Source } & \text { Reading } \\
\text { Intensity } & \\
\quad \text { Arbitrary units } \\
\end{array}$} & \multicolumn{2}{|c|}{$\begin{array}{l}\text { Percent } \\
\text { Transmission }\end{array}$} & $\begin{array}{l}\text { Temp. } \\
\text { Degrees F. }\end{array}$ \\
\hline \multicolumn{2}{|c|}{26 January. 1965} & \multicolumn{2}{|c|}{ No readings } & & & \\
\hline $\begin{array}{l}19 \text { Febru } \\
\text { Surface } \\
5 \\
10 \\
15 \\
20 \\
25 \\
30 \\
35 \\
40 \\
43.5 \\
40 \\
35 \\
30 \\
25 \\
20 \\
15 \\
10 \\
5 \\
\text { Surrace }\end{array}$ & $\begin{array}{l}\frac{1 y_{2} 1965}{00} \\
5 \\
10 \\
15 \\
20 \\
25 \\
30 \\
35 \\
40 \\
43 \\
40 \\
35 \\
30 \\
25 \\
20 \\
15 \\
10 \\
5 \\
00\end{array}$ & $\begin{array}{l}11.5 \\
11.5 \\
10.5 \\
11.5 \\
11.5 \\
11.0 \\
11.0 \\
11.4 \\
11.0 \\
11.4 \\
11.5 \\
11.5 \\
11.4 \\
11.5 \\
11.7 \\
11.4 \\
11.5 \\
10.4 \\
10.5\end{array}$ & $\begin{array}{l}5.1 \\
5.1 \\
4.8 \\
5.0 \\
5.5 \\
5.6 \\
6.0 \\
6.5 \\
6.5 \\
6.4 \\
6.5 \\
6.6 \\
6.1 \\
5.7 \\
5.4 \\
5.3 \\
5.4 \\
5.0 \\
4.7\end{array}$ & $\begin{array}{l}0.500 \\
0.443 \\
0.457 \\
0.435 \\
0.478 \\
0.508 \\
0.454 \\
0.571 \\
0.592 \\
0.562 \\
0.565 \\
0.574 \\
0.535 \\
0.496 \\
0.462 \\
0.465 \\
0.470 \\
0.482 \\
0.448\end{array}$ & $\begin{array}{l}0.69 \\
0.81 \\
0.78 \\
0.83 \\
0.74 \\
0.68 \\
0.79 \\
0.56 \\
0.52 \\
0.57 \\
0.57 \\
0.55 \\
0.62 \\
0.70 \\
0.77 \\
0.76 \\
0.75 \\
0.73 \\
0.80\end{array}$ & $\begin{array}{l}53.5 \\
53.5 \\
53.4 \\
53.2 \\
53.1 \\
53.0 \\
52.3 \\
52.0 \\
51.6 \\
51.5 \\
51.6 \\
52.0 \\
52.3 \\
53.0 \\
53.3 \\
53.2 \\
53.4 \\
53.5 \\
53.5\end{array}$ \\
\hline
\end{tabular}





\section{TABLE 3}

\section{LIGHT EXTINCTION DATA}

\begin{tabular}{|c|c|c|c|c|c|c|}
\hline $\begin{array}{l}\text { Meter } \\
\text { Wheel } \\
\text { Reading } \\
\text { Meters }\end{array}$ & $\begin{array}{l}\text { Accepted } \\
\text { Depth } \\
\text { Meters } \\
\end{array}$ & $\begin{array}{l}\text { Source } \\
\text { Light } \\
\text { Intensity } \\
\quad \text { Arb1.tr } \\
\end{array}$ & $\begin{array}{l}\text { Trans- } \\
\text { mittance } \\
\text { ary units }\end{array}$ & $\begin{array}{l}\text { Coefficiont } \\
\text { of } \\
\text { Extinction }\end{array}$ & $\begin{array}{l}\text { Temp. } \\
\text { OF }\end{array}$ & $\begin{array}{l}\text { Radiance } \\
\text { in } \% \text { of } \\
\text { Incldent } \\
\text { Sunlisht } \\
\end{array}$ \\
\hline \multicolumn{7}{|c|}{8 January, 1965} \\
\hline $\begin{array}{l}00 \\
5 \\
10 \\
15 \\
20 \\
25 \\
30 \\
35 \\
40 \\
45 \\
50 \\
55 \\
60 \\
65 \\
70 \\
75 \\
80 \\
85 \\
87\end{array}$ & $\begin{array}{l}00 \\
5 \\
10 \\
15 \\
20 \\
25 \\
30 \\
35 \\
40 \\
45 \\
50 \\
55 \\
60 \\
65 \\
70 \\
75 \\
80 \\
85 \\
87\end{array}$ & $\begin{array}{l}5.0 \times 10^{2} \\
5.5 \\
5.5 \\
5.5 \\
5.0 \\
5.0 \\
5.0 \\
5.0 \\
4.5 \\
4.5 \\
4.8 \\
5.0 \\
5.0 \\
5.0 \\
5.0 \\
5.0 \\
5.0 \\
5.0 \\
5.0\end{array}$ & $\begin{array}{l}800 \\
350 \\
220 \\
130 \\
70.0 \\
40.0 \\
35.0 \\
21.0 \\
12.0 \\
8.00 \\
6.00 \\
4.00 \\
2.50 \\
1.60 \\
1.00 \\
0.50 \\
0.20 \\
0.20 \\
0.0\end{array}$ & $\begin{array}{l}1.6 \\
0.637 \\
0.400 \\
0.236 \\
0.140 \\
0.080 \\
0.070 \\
0.042 \\
0.0266 \\
0.0178 \\
0.0125 \\
0.0080 \\
0.0050 \\
0.0032 \\
0.0020 \\
0.0010 \\
0.0004 \\
0.0004 \\
0.000\end{array}$ & $\begin{array}{l}54.4 \\
54.2 \\
54.2 \\
54.2 \\
54.2 \\
54.3 \\
54.4 \\
54.8 \\
54.8 \\
54.8 \\
54.7 \\
54.9 \\
54.2 \\
54.7 \\
53.1 \\
52.8 \\
52.3 \\
52.1 \\
52.0\end{array}$ & $\begin{array}{c}100 \\
39.8 \\
25.0 \\
14.7 \\
8.8 \\
5.0 \\
4.4 \\
2.6 \\
1.7 \\
1.1 \\
0.8 \\
0.5 \\
0.3 \\
0.2 \\
0.1 \\
0.1 \\
0.025 \\
0.025 \\
0\end{array}$ \\
\hline $\begin{array}{l}00 \\
10 \\
20 \\
30 \\
40 \\
50 \\
60 \\
70 \\
80 \\
87\end{array}$ & $\begin{array}{l}00 \\
10 \\
20 \\
30 \\
40 \\
50 \\
60 \\
70 \\
80 \\
87\end{array}$ & $\begin{array}{l}5.0 \\
4.8 \\
4.3 \\
4.4 \\
5.0 \\
5.0 \\
5.0 \\
5.0 \\
5.0 \\
5.0\end{array}$ & $\begin{array}{c}750 \\
220 \\
86.0 \\
30.0 \\
14.0 \\
6.50 \\
2.50 \\
1.00 \\
0.20 \\
0.0\end{array}$ & $\begin{array}{l}1.5 \\
0.458 \\
0.200 \\
0.0682 \\
0.0280 \\
0.0130 \\
0.0050 \\
0.0020 \\
0.0008 \\
0.000\end{array}$ & $\begin{array}{l}54.4 \\
54.2 \\
54.2 \\
54.4 \\
54.18 \\
54.7 \\
54.2 \\
53.1 \\
52.3 \\
52.0\end{array}$ & $\begin{array}{r}100 \\
32.4 \\
13.3 \\
4.5 \\
1.9 \\
0.9 \\
0.3 \\
0.1 \\
0.1 \\
0.0\end{array}$ \\
\hline
\end{tabular}


TABLE 3

Meter Accepted Source Transo

Wheel

Reading

Meters

Depth

Meters

26 January, 1965

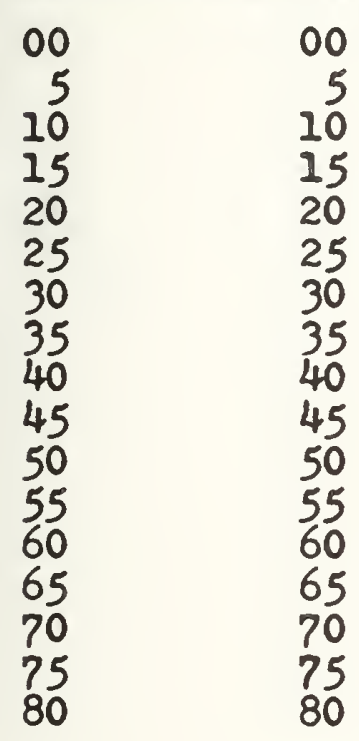

$00 \quad 00$

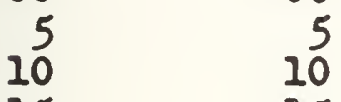

$15 \quad 15$

20

25

30

35

45

50

55

65

75

77
00

10

15

25

35

45

50

60

65

75

80

5

15
20

25

30

35
40

45

50

55
60

65

70

77
Iight

Intensity

mittance
Coefficient Temp. of

Extinction

OF Arbitrary units

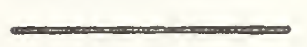

Radlance in $\%$ of Incident Sunl1ght
$8.0 \times 10^{2}$

8.0

8.0

8.0

8.0

8.0

8.0

8.0

8.0

8.0

8.0

8.0

8.0

8.0

9.0

8.5

8.5

8.0

8.0

8.0

8.0

8.0

8.0

7.5

7.0

7.0

7.0

$7 \cdot 5$

8.5

9.0

9.0

9.0

8.0
1200
400
200
no

no reading

54.0

26.0

15.0

9.00

5.30

3.50

2.20

1. 50

1.00

0.50

0.40

0.20

0.20

1000

550

350

123

59.0

30.0

15.0

8.80

5.60

3.50

2.40

1.50

1.00

0.60

0.40

0.40
1.50

0.500

0.250

0.0675

0.0325

0.01875

0.01125

0.00663

0.00438

0.00275

0.001875

0.001250

0.000625

0.000445

0.000235

0.000235

1.25

0.693

0.425

0.154

0.0738

0.0375

0.0200

0.0126

0.00800

0.00500

0.00320

0.001765

0.001111

0.000667

0.000445

0.000500
54.1

54.0

54.0

54.0

53.9

53.9

53.8

53.6

53.2

52.8

52.2

51.6

51.1

51.0

50.7

50.5

50.4

54.1

54.0

54.0

54.0

53.9

53.9

53.8

53.6

53.2

52.8

52.2

51.6

51.1

51.0

50.7

50.4

$$
\begin{aligned}
& 100 \\
& 33.3 \\
& 16.7 \\
& \infty \\
& 4.50 \\
& 2.20 \\
& 1.30 \\
& 0.80 \\
& 0.40 \\
& 0.25 \\
& 0.2 \\
& 0.1 \\
& 0.1 \\
& 0.04 \\
& 0.03 \\
& 0.01
\end{aligned}
$$

100

55.4

34.0

12.3

5.8

3.0

2.6

1.0

0.64

0.40

0.25

0.14

0.09

0.05

0.03

\section{Pebruary, 1965}

$\begin{array}{rrrrrrr}00 & 00 & 6.9 & 480 & 0.695 & 53.5 & 100 \\ 5 & 5 & 6.5 & 320 & 0.492 & 53.5 & 71 \\ 10 & 10 & 6.0 & 160 & 0.267 & 53.4 & 38.6 \\ 15 & 15 & 7.3 & 70.0 & 0.096 & 53.2 & 13.8 \\ 20 & 20 & 8.0 & 12.0 & 0.0150 & 53.3 & 2.2 \\ 24.5 & 24 & 8.0 & 0.0 & 0.0 & 53.0 & 0\end{array}$


TABLE 3

\begin{tabular}{|c|c|c|c|c|c|c|}
\hline $\begin{array}{l}\text { Meter } \\
\text { Wheel } \\
\text { Reading } \\
\text { Meters } \\
\end{array}$ & $\begin{array}{l}\text { Accepted } \\
\text { Depth } \\
\text { Meters }\end{array}$ & $\begin{array}{l}\text { Source } \\
\text { Ilght } \\
\text { Intensity } \\
\quad \text { Arb1tr } \\
\end{array}$ & $\begin{array}{l}\text { Trans- } \\
\text { mittance } \\
\text { ry units }\end{array}$ & $\begin{array}{l}\text { Coefficiont } \\
\text { of } \\
\text { Extinction }\end{array}$ & $\begin{array}{l}\text { Temp. } \\
\text { OF }\end{array}$ & $\begin{array}{l}\text { Radiance } \\
\text { In } 8 \text { of } \\
\text { Incident } \\
\text { Sunlight }\end{array}$ \\
\hline \multicolumn{7}{|c|}{19 February, 1965} \\
\hline $\begin{array}{l}00 \\
5 \\
10 \\
15 \\
20 \\
24.5\end{array}$ & $\begin{array}{r}00 \\
5 \\
10 \\
15 \\
20 \\
24\end{array}$ & $\begin{array}{l}8.0 \\
7.0 \\
5.8 \\
5.5 \\
6.5 \\
8.0\end{array}$ & $\begin{array}{c}700 \\
300 \\
182 \\
86.0 \\
9.50 \\
0.0\end{array}$ & $\begin{array}{l}0.875 \\
0.425 \\
0.314 \\
0.156 \\
0.0146 \\
0.0\end{array}$ & $\begin{array}{l}53.5 \\
53.5 \\
53.4 \\
53.2 \\
53.3 \\
53.0\end{array}$ & $\begin{array}{c}100 \\
48.6 \\
35.9 \\
17.8 \\
1.7 \\
0\end{array}$ \\
\hline
\end{tabular}



TABLE 4

Scattering Coefficient, Density, Phosphate, Particle size and Concentration

Sample Field Work Depth Phosphate Temp. SaIInIty Density Number

\section{Date}
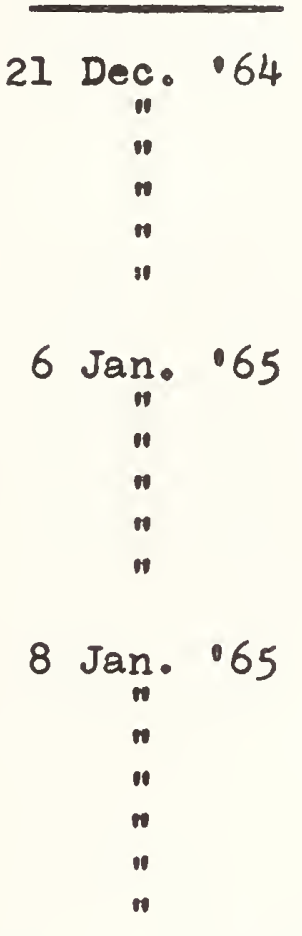

19

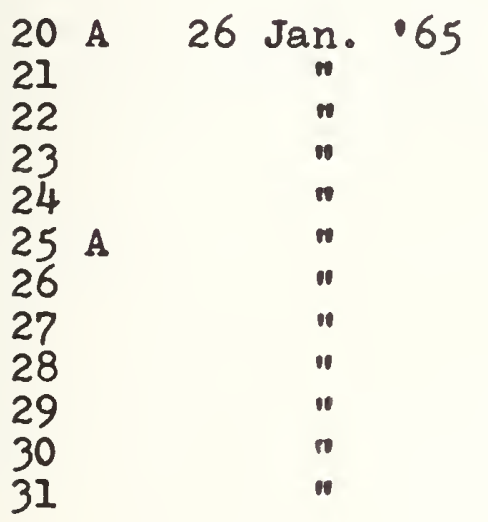

\section{Meters}

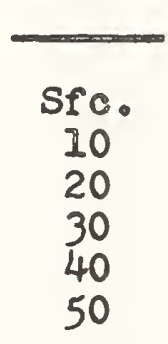

Sfe.

25

75

100

125

Sfo.

30
100

150

200

250

300

sfe.

10

20

30

40

50

75

100

150

200

250

300

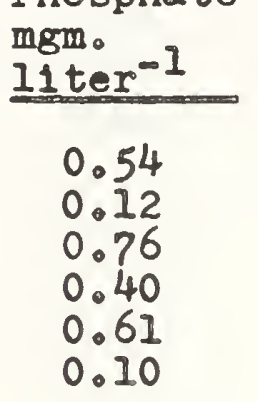

0.11

0.12

0.08

0.18

0.10

cloudy

0.48

0.71

0.46

0.78

0.98

0.97

1.05

0.10

0.28

0.28

0.28

0,96

0.73

0.73

0.60

$\left(\mathrm{N} . \mathrm{C}_{2}\right)+0.41^{4}$

0.59

0.78

0.96
Degrees $0 / 100$

C.

$\begin{array}{ll}12.8 & 33.074 \\ 12.1 & 33.162 \\ 11.8 & 33.180 \\ 11.7 & 33.290 \\ 11.6 & 33.416 \\ 11.5 & 33.332\end{array}$

12.2

11.7

11.9

12.0

12.0

11.1

33.234

33.304

33.364

33.356

33.117

33.420

$12.3 \quad 32.784$

$12.4 \quad 33.197$

10.3

$9 \cdot 3$

33.695

33.859

33.912

7.8

6.9

33.920

34.078

$12.3 \quad 33.315$

$12.8 \quad 33.372$

$12.2 \quad 33.378$

12.133 .638

11.8

33.742

11.2

10.3

10.1

9.3
8.8

8.1

7.6
33.822

33.639

34.213

34.061

34.212

34.263
33.071 $\sigma_{t}$

24.960

25.160

25.230

25.340

25.450

25.410

25.210

25.350

25.360

25.330

25.150

25.550

24.830

25.130

25.900

26.200

26.350

26.480

26.731

25.250

25.190

25.310

25.530

25.670

25.840

25.860

26.230

26.470

26.440

26.660

26.770 

TABLE 4

\begin{tabular}{|c|c|c|c|c|c|c|c|c|c|c|}
\hline $\begin{array}{l}\text { ample } \\
\text { umber }\end{array}$ & & $\begin{array}{l}\text { eld } \\
\text { ite }\end{array}$ & Work & $\begin{array}{l}\text { Depth } \\
\text { Meters }\end{array}$ & $\begin{array}{l}\mathrm{P} \\
\mathrm{m} \\
1\end{array}$ & $\begin{array}{l}\text { Phosphe } \\
\text { mgmo } \\
\text { liter }\end{array}$ & & $\begin{array}{l}\text { Temp。 } \\
\text { Degrees } \\
\text { C. }\end{array}$ & $\begin{array}{l}\text { Salinity } \\
\% / 00\end{array}$ & $\begin{array}{c}\text { Dens1ty } \\
\sigma_{t}\end{array}$ \\
\hline $\begin{array}{l}32 \\
33 \\
34 \\
35 \\
36 A \\
37 \\
38 B \\
39 \\
40 \\
41 \\
42 \\
43 \\
44\end{array}$ & 19 & $\begin{array}{c}\text { Feb. } \\
" \\
" 1 \\
" \\
" \\
" \\
" \\
" \\
"\end{array}$ & .65 & $\begin{array}{l}\text { Sfe. } \\
10 \\
20 \\
17 \cdot 5 \\
30 \\
40 \\
30 \\
43 \\
54 \\
75 \\
92 \\
105 \\
110\end{array}$ & $\begin{array}{l}\left(b \circ t_{0}\right) \\
\left(b \circ t_{0}\right)\end{array}$ & $\begin{array}{l}0.14 \\
0.35 \\
0.30 \\
0.43 \\
0.42 \\
0.59 \\
0.51 \\
0.83 \\
0.32 \\
0.71 \\
0.52 \\
4.70 \\
2.35\end{array}$ & . & $\begin{array}{l}12.0 \\
11.9 \\
11.8 \\
12.0 \\
11.3 \\
11.1 \\
11.3 \\
10.8 \\
11.0 \\
10.9 \\
10.6 \\
10.5 \\
10.6\end{array}$ & $\begin{array}{l}33.476 \\
33.468 \\
33.422 \\
33.467 \\
33.569 \\
33.582 \\
33.665 \\
33.702 \\
33.477 \\
33.506 \\
33.561 \\
33.826 \\
33.993\end{array}$ & $\begin{array}{l}25.430 \\
25.440 \\
25.420 \\
25.420 \\
25.630 \\
25.670 \\
25.700 \\
25.820 \\
25.610 \\
25.650 \\
25.750 \\
25.970 \\
26.080\end{array}$ \\
\hline $\begin{array}{ll}45 & B \\
46 & B\end{array}$ & 26 & $\underset{*}{\operatorname{Jan}}$ & .65 & $\begin{array}{c}\text { Sfc. } \\
50\end{array}$ & & $\begin{array}{l}\text { Short } \\
\text { Short }\end{array}$ & **** & $\begin{array}{l}12.3 \\
11.2\end{array}$ & $\begin{array}{l}\text { Short } \\
\text { Short }\end{array}$ & $\infty-\infty$ \\
\hline
\end{tabular}

* (N. C.) Not conclusive, solution turned yellow.

* (bot.) Bottle contained sediment.

** Short Not sufficient sample avallable to perform analysis. 

TABLE 4
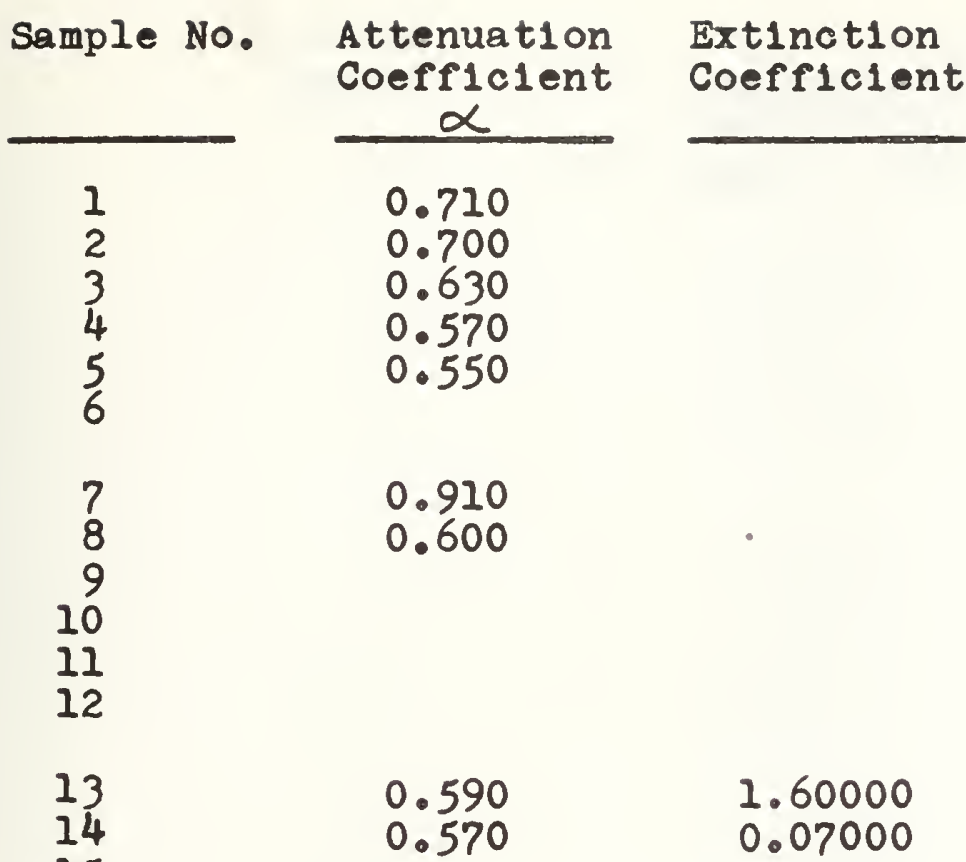

0.590

14

15
16

17

18

19

$20 \mathrm{~A}$

21

22

23

24

25

27

28

29

30

31

32

33

35

36 A

37

38 B

39

40

41

42

43

44

$45 \mathrm{~B}$

$46 \mathrm{~B}$
0.690

0.780

0.740

0.830

0.790

0.520

0.620

0.570
1.50000

0.25000

0.06750

0.01875

0.00663

0.00275

0.00023

0.69500

0.26700

0.01500

0.09600

1.50000

0.00275
$B=\frac{2 \pi r}{\lambda}$

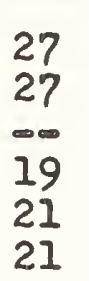

19.6

26

26

32

$\infty$

$\infty$

32

32

22

22

22

22

26

17

22

22

22

27

17

27

22

16

22

16

22

21

5

21

13

5

$27 \cdot 5$

22.0

30

21

27

32

8

21

25
Particle

Mean

$\operatorname{Rad1us}(r)$

(microns)

2.36

$-\infty 000$

1.66

1.84

1.84

1. 72

2.28

2.80

$\infty 0 \infty$

$\infty 000$

2.80

2.80

1.93

1.93

1.93

1.93

2.28

1.99

1.93

1.93

1.93

2.36

1.49

2.36

1.93

1.41

1.93

1.41

1.93

1.84

0.49

1.84

1.11

0.44

0.44

2.35

1.88

2.64

1. 84

2.36

2. 80

0.70

1.79

2.13
Relative

Refractive

Index (m)

1.26

1.26

$-\infty \infty \infty$

1.26

1.26

1.26

1.26

1.33

1.33

1.25

$-\infty \infty 0$

$\infty \infty \infty$

1.25

1.25

1.33

1.33

1.33

1.33

1.26

1.26

1.25

1.25

1.25

1.33

1. 26

1.26

1.33

1.33

1.25

1.26

1.25

1.25

1.25

1.25

1.24

1.25

1.25

1.33

1.33

1.20

1.25

1.26

1. 33

1.25

1.33

2. 33 

TABLE 4

Samplo

Number

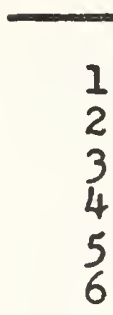

7
8
9
10
11
12

13

14

15

16

17

18

19

$20 \mathrm{~A}$

21

22

23

24

25

26

27

28

29

30

31

32

33

34

35

36 A

37

38 B

39

40

41

42

43

44
Volume

scattering Coeffielent

Meter-1 (S)

0.2179

0.3123

Short

0.2367

0.1794

0.4018

0.6466

0.8401

1.2020

0.4194

Short

Short

0.7649

0.4463

0.3714

0.1301

0.1778

0.3078

0.2288

0.9990

0.9646

0.9658

0.9579

0.9664

1.0010

0.0917

0.9252

0.0960

0.8854

0.8512

0.9271

0.3642

0.3456

0.3286

0.3332

0.3317

0.3363

0.3315

0.3379

0.3919

0.3109

0.3484

0.4143

0.5113
Concentration

Partl $1 \mathrm{c} \frac{1}{3}$ es
Per cm

$$
3 . \frac{11}{4.46}
$$

10.3

6.04

13.5

27.5

15.4

22.1

10.0

$-\infty$

8. 32

5.40

13.3

4.63

6.33

11.0

3.62

44.8

25.8

25.8

25.6

16.6

44.9

1.57

23.7

4.27

21.0

42.6

24.8

10.7

202

11.0

17.5

194

197

$$
\begin{gathered}
5.53 \\
12.7 \\
3.35 \\
10.4 \\
4.87 \\
3.53 \\
7.15
\end{gathered}
$$



TABLE 4

Sample Number

$45 \mathrm{~B}$

$46 \mathrm{~B}$
Volume

Scattering Coerficlent

Meter-1 (5)

0.9491

1.0031
Concentration Particles $\mathrm{cm}^{3} \times \mathrm{xO}^{4}$

39.2

21.9 

TABLE 5

MEASURED SCATTERING INTENSITY DATA

Angle

$\begin{array}{llr}179.00 & 7 * & 309.95671 \\ 170.00 & & 20.64516 \\ 160.00 & & 29.99999 \\ 150.00 & & 25.71429 \\ 140.00 & & 26.66667 \\ 130.00 & & 24.54545 \\ 120.00 & & 20.00000 \\ & 6 & \end{array}$

179.00

170.00

160.00

150.00

140.00

130.00

179.00

170.00

160.00

150.00

140.00

130.00

179.00

170.00

160.00

150.00

140.00

130.00

120.00

179.00

170.00

160.00

150.00

140.00

130.00

120.00

179.00

170.00

160.00

150.00

140.00

130.00
Sigma theta

311.68831

29.03226

45.55556

45.71429

44.99999

32.72727

6

303.03030

46.12903

35.55556

29.28571

29.16667

24.54545

7

$265.8008 ?$

29.03226

45.55556

38.57143

60.00000

16.36363

10.00000

7

309.95671

29.03226

29.99999

32.14286

30.00000

74.54545

6

40.00000

366.23377

56.12903

57.77778

46.42858

62.50000

79.09091
Logsigtheta Sample

1

5.73643

3.02748

3.40120

3.24705

3.28341

3.20053

2.99573

2

5.74200

3.36841

3.81893

3.82241

3.80666

3.48821

5.71383

3.83144

3.57110

3.57710

3.37303

3.20053

5.58275

3.36841

3.81893

3.65251

4.09434

2.79506

2.30259

5.73643

3. 36841

3.40120

3.47019

3.40120

4. 31141

3.68888

5.90327

4.02765

4.05660

3.83792

4.13517

4.37060
6

4

5

* Number of data points for each sample. 

TABLE 5

Angle

179.00

170.00

160.00

150.00

140.00

130.00

179.00

170.00

160.00

150.00

140.00

130.00

179.00

170.00

160.00

150.00

140.00

130.00

179.00

170.00

160.00

150.00

140.00

130.00

120.00

179.00

170.00

160.00

150.00

130.00

179.00

170.00

160.00

150.00

140.00

130.00

179.00

170.00

160.00

150.00

140.00
Sigma the ta

6

6

300.86580

119.03226

193.33333

232.85714

108.33333

79.09091

348.48485

140.32258

241.66667

232.85714

217.50000

6

118.18182

309.95671

56.12903

84.44444

92.85714

72.50000

39.09091

7

290.90909

72.25806

101.11111

100.00000

81.66667

76.36364

77.77778

5

261.03896

58.70968

78.88889

40.00000

6

50.90909

290.90909

45.16129

62.22222

70.00000

46.66667

38.18182

5

261.30896

36.12903

46.66667

30.00000

11.66667
Logsigtheta Sample

8

5.70666

4.77939

5.26442

5.45043

4.68521

4.37060

5.85359

4.94394

5.48756

5.45043

5.38220

4. 77222

5.73643

4.02765

4.43609

4.53106

4.28359

3.66589

5.67301

4.28024

4.61622

4.60517

4.40265

4. 33551

4.35386

5.56467

4.07260

4.36804

3.68888

3.93004

5.67301

3.81024

4.13071

4.24850

3.84303

3.64236

10

13

14

15

5.56570

3.58710

3.84303

3.40120

2.45674 

TABLE 5

Angle

179.00

170.00

160.00

150.00

140.00

130.00

179.00

170.00

160.00

150.00

140.00

179.00

170.00

160.00

150.00

140.00

179.00

178.00

177.00

176.00

175.00

174.00

173.00

172.00

171.00

170.00

160.00

150.00

140.00

130.00

120.00

110.00

100.00

90.00
Sigma theta

6

$$
\begin{array}{r}
273.16017 \\
40.32258 \\
54.44444 \\
50.00000 \\
35.00000 \\
12.72727
\end{array}
$$

5

254.97835

45.80645

62.22222

59.99999

35.00000

5

139.39394

49.67742

69.44444

59.99999

23.33333

18

184.09425

85.79410

61:34970

51.02040

35.17315

22.04970

20.71430

21.17940

20.00000

20.97505

30.13395

42.20780

54.62185

61.90475

71.42855

77.38095

81.16885

92.85710
Logsigtheta Sample

5.61006

3.69691

3.99718

3.91202

3.55535

2.54375

5.54118

3.82442

4.13071

4.09434

3.55535

4.93730

3.90555

4.24053

4.09434

3.14988

5.21545

4.45195

4.11659

3.93223

3.56028

3.09330

3.03082

3.05303

2.99573

3.04333

3.40565

3.74261

4.00043

4.12560

4. 26870

4.34874

4.39653

4.53106
17

18

19

20 

TABLE 5

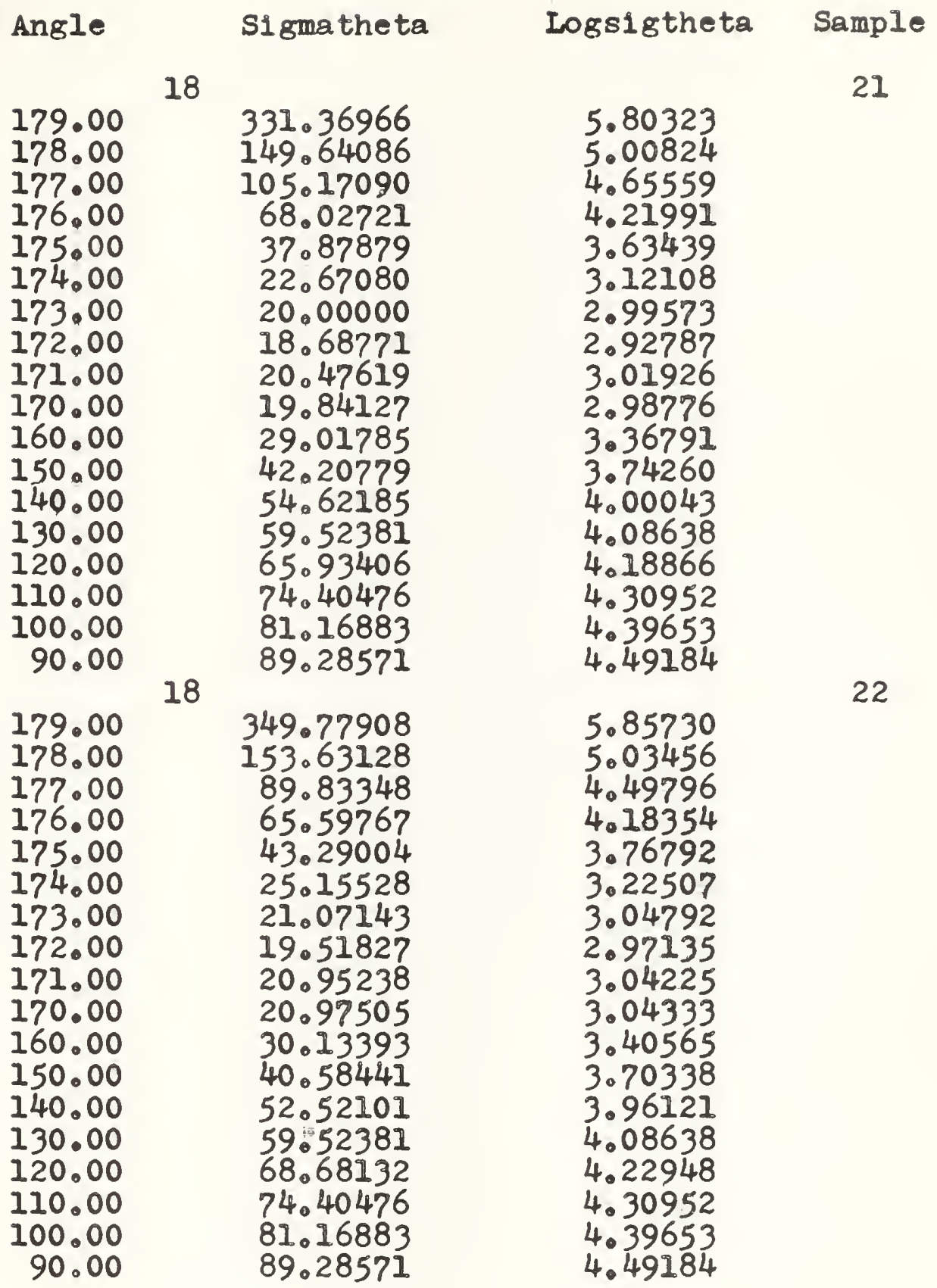





\section{TABLE 5}

Angle

179.00

178.00

177.00

176.00

175.00

174.00

173.00

172.00

171.00

170.00

160.00

150.00

140.00

130.00

120.00

110.00

100.00

90.00

179.00

178.00

177.00

176.00

175.00

174.00

173.00

172.00

171.00

170.00

160.00

150.00

140.00

130.00

120.00

110.00

100.00

90.00
Sigma theta

18

121.50221

75.81803

59.15863

46.16132

29.76190

18.63354

15.71428

15.78073

16.19047

18.14059

29.01785

40.58441

52.52101

58.33333

67.30769

71.42857

81.16883

89.28571

18

865.24300

678.37190

372.48028

160.34985

70.34632

34.16149

18.92857

17.02658

18.57143

19.27437

19.01785

40.58441

52.52101

59.52381

68.68132

74.40476

81.16883

89.28571
Logsigtheta Samplo

23

4.79993

4. 32834

4.08022

3.83214

3.39323

2.92496

2.75457

2.75879

2.78442

2.89815

3.36791

3.70338

3.96121

4.06617

4.20927

4.26870

4.39653

4.49184

6.76301

6.51970

5.92018

5.07736

4.25343

3.53110

2.94067

2. 83478

2.92162

2.95878

2.94538

3.70338

3.96121

4.08638

4.22948

4.30952

4.39653

4.49184 

TABLE 5

Angle

179.00

178.00

177.00

176.00

175.00

174.00

173.00

172.00

171.00

170.00

160.00

150.00

140.00

130.00

120.00

110.00

100.00

90.00

179.00

178.00

177.00

176.00

175.00

174.00

173.00

172.00

171.00

170.00

160.00

150.00

140.00

130.00

120.00

110.00

100.00

90.00
S1gma theta

18

77.31959

57.86113

56.96757

38.87269

27.05627

19.56521

16.42857

15.78073

16.66666

18.70748

30.13393

42.20779

54.62185

61.90476

71.42857

77.38095

84.41558

92.85714

18

184.09425

155.62649

127.08150

97.18173

67.64069

34.16149

20.71428

?. 48848

7.50682

6.79228

7.84632

6.73302

?.02075

7.14285

7.26928

7.49362

7.40025

7.46753
Logsigtheta Sample

25

4. 34795

4.05805

4. 04248

3.66029

3.29792

2.97375

2.79902

2.75879

2.81341

2.92892

3.40565

3.74260

4.00043

4.12560

4.26870

4. 34874

4.43575

4.53106

5.21545

5.04746

4.84483

4.57658

4.21421

3.53110

3.03082

2.0133 ?

2.01581

1.91579

2.06004

1.90702

1.94887

1.96611

1.98366

2.01405

2.00151

2.01056 

TABLE 5

Angle

18

179.00

178.00

177.00

176.00

175.00

174.00

173.00

172.00

171.00

170.00

160.00

150.00

140.00

130.00

120.00

110.00

100.00

90.00

179.00

178.00

177.00

176.00

175.00

174.00

173.00

172.00

171.00

170.00

160.00

150.00

140.00

130.00

120.00

110.00

100.00

90.00

18
Sigma theta

$$
\begin{array}{r}
38.65979 \\
25.93774 \\
12.48904 \\
8.50340 \\
4.32900 \\
2.23602 \\
1.60714 \\
1.49501 \\
1.61904 \\
1.75737 \\
2.79018 \\
3.89610 \\
4.83193 \\
5.47619 \\
6.59340 \\
6.84523 \\
7.46753 \\
8.57142
\end{array}
$$

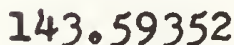

109.73663

59.21560

70.30675

37.87879

19.25466

14.64285

14.95016

14.76190

17.00680

27.90178

38.96104

50.42017

$57 \div 14285$

65.93406

71.42857

77.92208

85.71428
Logsigtheta Sample

27

3.65480
3.25570
2.52485
2.14047
1.46534
.80470
.47446
.40214
.48184
.56382
1.02611
1.35998
1.57525
1.70041
1.88607
1.92355
2.01056
2.14843

4.96699

4.69808

4.08119

4.25287

3.63439

2.95775

2.68395

2.70472

2.69205

2.83361

3.32869

3.66256

3.92039

4.04555

4.18866

4.26870

4.35571

4.45102 
TABLE 5

Angle

179.00

178.00

177.00

176.00

175.00

174.00

173.00

172.00

171.00

170.00

160.00

150.00

140.00

130.00

120.00

110.00

100.00

90.00

179.00

178.00

177.00

176.00

175.00

174.00

173.00

172.00

171.00

170.00

160.00

150.00

140.00

130.00

120.00

110.00

100.00

90.00
Sigma theta

18

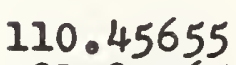

81.80365

89.83345

65.59765

47.46755

18.94410

14.64285

14.11960

15.23805

17.00680

26.78570

37.33765

48.31935

54.76190

63.18680

66.96430

74.67530

18

82.14285

607.51104

438.94653

262.92725

116.61807

51.40692

22.67080

16.07143

15.36545

15.23809

16.43991

25.11160

35.71428

45.16806

52.38095

60.43956

65.47619

71.42857

78.57143
Logsigtheta Sample

4.70462

4.40432

4.49796

4.18354

3.86005

2.94149

2.68395

2.64756

2.72380

2. 83361

3.28787

3.62000

3.87783

4.00299

4.14610

4.20416

4. 31315

4.40846

6.40937

6.08438

5.57188

4.75890

3.93977

3.12108

2.77704

2.73212

2.72380

2.79971

3.22333

3.57555

3.81039

3.95854

4.10164

4.18169

4.26870

4.36401 

TABLE 5

Angle

18

179.00

178.00

177.00

176.00

175.00

174.00

173.00

172.00

171.00

170.00

160.00

150.00

140.00

130.00

120.00

110.00

100.00

90.00

18

179.00

178.00

177.00

176.00

175.00

174.00

173.00

172.00

171.00

170.00

160.00

150.00

140.00

130.00

120.00

110.00

100.00

90.00
S1gma theta

Logsigtheta Sample

4.73741

4.40432

4.18558

3.93223

3.39323

2.85597

2.55390

2.58694

2.69205

2.79971

3.32869

3.70338

3.96121

4.04555

4.18866

4.26870

4.35571

4.45102

6.27915

4.23807

4.07577

3.55920

3.09654

2.81515

2.69463

2.53037

2.46243

2.37896

2.62104

2.99573

3.25355

3.33220

3.47531

3.30811

3.38285

3.38285 

TABLE 5

Angle

$\begin{array}{rrr} & 18 & \\ 179.00 & & 984.61538 \\ 178.00 & & 102.79329 \\ 177.00 & & 61.34969 \\ 176.00 & & 43.24324 \\ 175.00 & & 23.93939 \\ 174.00 & 17.39130 \\ 173.00 & 13.20000 \\ 172.00 & 11.62790 \\ 171.00 & 10.66666 \\ 170.00 & 10.79365 \\ 160.00 & 15.00000 \\ 150.00 & 13.18181 \\ 140.00 & & 18.82352 \\ 130.00 & & 21.60000 \\ 120.00 & & 25.23076 \\ 110.00 & & 27.00000 \\ 100.00 & & 29.09090 \\ 90.00 & & 31.27272 \\ 18 & & \end{array}$

179.00

178.00

177.00

176.00

175.00

174.00

173.00

172.00

171.00

170.00

160.00

150.00

140.00

130.00

120.00

110.00

100.00

90.00
Sigma theta

984.61538

61.34969

43.24324

23.93939

13.20000

11.62790

10.66666

15.00000

13.18181

18.82352

21.60000

27.00000

29.09090

18

$574.3589 ?$

53.63128

44.17177

10.13513

18.18181

13.21739

10.79999

9.06666

9.52380

12.50000

14.72727

19.05882

21.60000

24.92307

27.00000

28.72727

29.09090
9.76744
Logsigtheta Sample

33

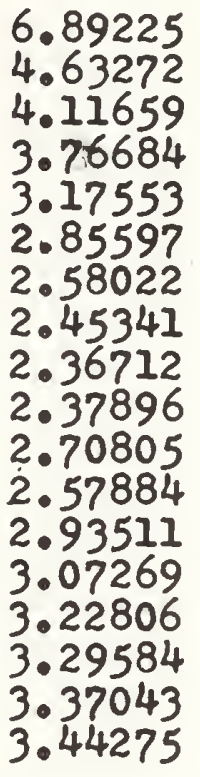

6.35325

3.98213

3.78809

2. 31601

2. 90042

2.58153

2. 37955

2.27905

2.20460

2.25379

2.52573

2.68970

2. 94753

3.07269

3.21579

3.29584

3.35785

3. 37043 

TABLE 5

Angle

179.00

178.00

177.00

176.00

175.00

174.00

173.00

172.00

171.00

170.00

160.00

150.00

140.00

130.00

120.00

110.00

100.00

90.00

179.00

178.00

177.00

176.00

175.00

174.00

173.00

172.00

171.00

170.00

160.00

150.00

140.00

130.00

120.00

110.00

100.00

90.00
Sigma the ta

18

861.53846

71.50837

49.07975

35.13513

19.39393

15.65217

14.40000

13.02325

9.60000

10.15873

10.75000

15.09090

19.05882

21.33333

25.23076

27.33333

29.81818

18

29.45454

779.48717

71.50837

46.62576

37.83783

22.42424

14.95652

12.00000

10.69767

9.60000

10.15873

10.75000

14.90909

19.29411

21.60000

24.30769

27.00000

29.45454

29.45454
Logsigthe ta Sample

35

6.75872

4.26981

3.89345

3.55920

2.96496

2.75061

2.66723

2.56674

2. 26176

2. 31833

2. 37491

2.71409

2.94753

3.06027

3.22806

3.30811

3.39512

3.38285

6.65864

4.26981

3.84215

3.63331

3.11014

2.70515

2.48491

2. 37003

2.26176

2. 31833

2. 37491

2.70197

2.95980

3.07269

3.19079

3.29584

3.38285

3.38285 

TABLE 5

Angle

179.00

178.00

177.00

176.00

175.00

174.00

173.00

172.00

171.00

170.00

160.00

150.00

140.00

130.00

120.00

110.00

100.00

90.00

179.00

178.00

177.00

176.00

175.00

174.00

173.00

172.00

171.00

170.00

160.00

150.00

140.00

130.00

120.00

110.00

100.00

90.00
S1 gma the ta

18

$$
\begin{array}{r}
1194.23076 \\
151.95530 \\
107.97546 \\
83.78378 \\
60.60606 \\
45.21739 \\
31.19999 \\
26.04651 \\
24.00000 \\
23.49456 \\
16.25000 \\
18.18181 \\
19.29411 \\
21.33333 \\
24.61538 \\
26.33333 \\
28.36363 \\
29.09090
\end{array}
$$

18

1169.23077

105.02793

51.53374

37.83784

24.84848

16.34782

12.40000

10.69767

10.13333

10.15873

10.87500

14.54545

19.05882

21.33333

24.61538

26.66666

29.09091

29.45454
Logsigtheta Sample

7.08526

37

5.02359

4.68190

4.42824

4.10439

3.81148

3.44042

3.25988

3.17805

3.15677

2.78809

2.90042

2.95980

3.06027

3.20337

3.27084

3. 34511

3.37043

7.06410

38

4.65423

3.94224

3.63331

3.21280

2.79409

2. 51770

2.37003

2. 31583

2.31833

2. 38647

2.67728

2. 94753

3.06027

3.20337

3.28341

3.37043

3.38285 

TABLE 5

Angle

179.00

178.00

177.00

176.00

175.00

174.00

173.00

172.00

171.00

170.00

160.00

150.00

140.00

130.00

120.00

110.00

100.00

90.00

179.00

178.00

177.00

176.00

175.00

174.00

173.00

172.00

171.00

170.00

160.00

150.00

140.00

130.00

120.00

110.00

100.00

90.00
Sigma theta

18

1374.35897

71.50838

58.89570

37.83784

22.72727

17.04348

14.00000

11.62790

11.20000

11.42857

10.75000

15.27272

19.52941

21.86666

25.53846

27.33333

29.81818

29.81818

18

1600.00000

160.89385

56.44172

45.94594

23.03030

17.04348

14.40000

12.09302

11.20000

11.42857

15.00000

15.45454

19.52941

22.40000

25.53846

27.66666

30.54545

29.81818
Logsigtheta Sample

39

7.22574

4.26981

4.07577

3.63331

3.12357

2.83577

2.63906

2.45341

2.41591

2.43612

2. 37491

2.72607

2.97192

3.08496

3.24019

3.30811

3.39512

3.39512

7. 37776

5.08074

4.03321

3.82747

3.13681

2.83577

2.66723

2.49263

2.41591

2.43612

2. 70805

2.73790

2.97192

3.10906

3.24019

3.32023

3.41922

3.39512 

TABLE 5

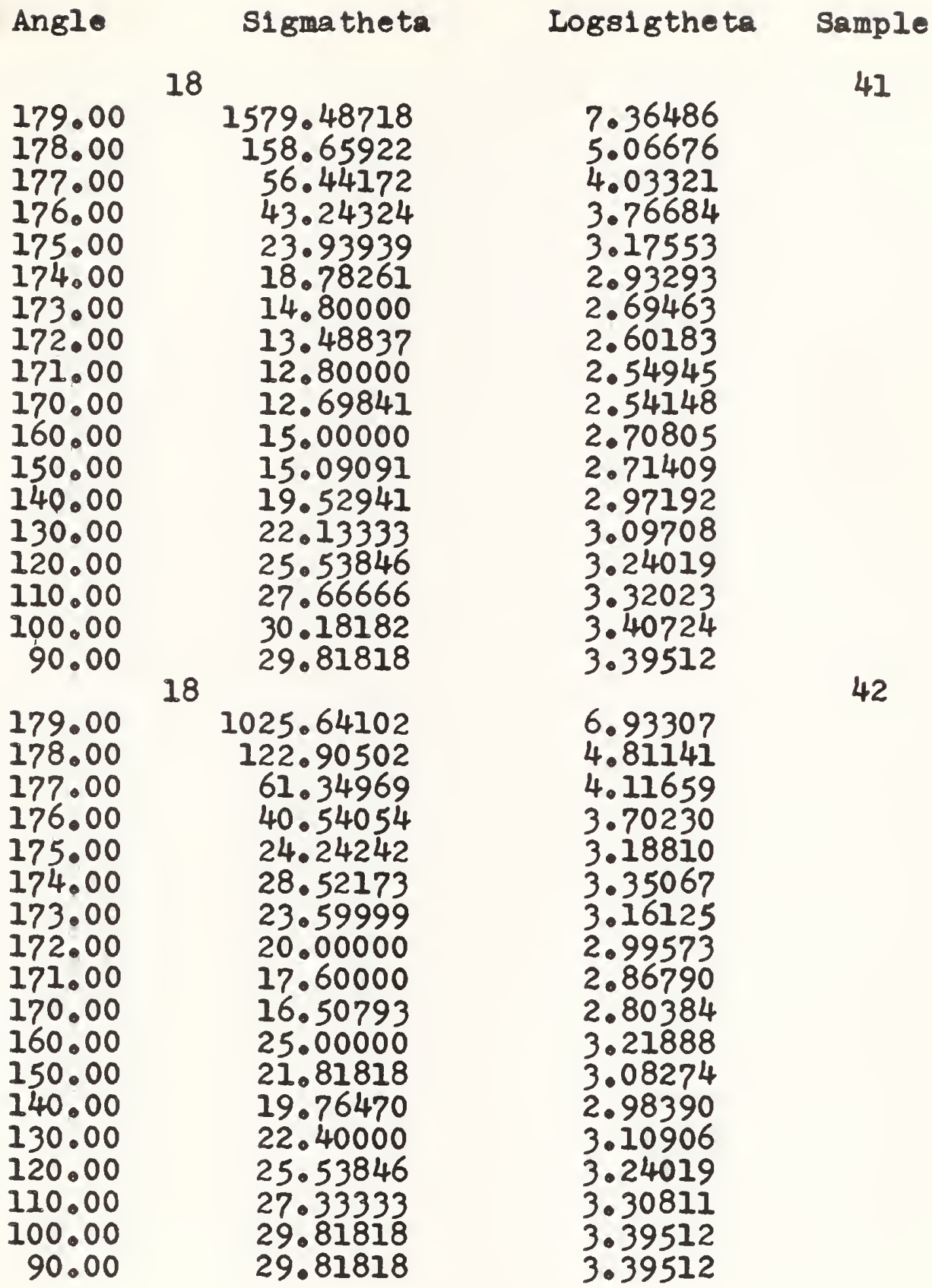



TABLE 5

Angle

179.00

178.00

177.00

176.00

175.00

174.00

173.00

172.00

171.00

170.00

160.00

150.00

140.00

130.00

120.00

110.00

100.00

90.00

179.00

178.00

177.00

176.00

175.00

174.00

173.00

172.00

171.00

170.00

160.00

150.00

140.00

130.00

120.00

110.00

100.00

90.00
S1 gma theta

18

6.97435

7.59776

8.09815

8.64864

9.69696

11.13043

12.40000

13.95348

15.46666

17.77777

26.25000

30.90909

35.29411

34.66666

36.92307

40.00000

33.09090

32.00000

18

615.38461

491.62011

392.63803

351.35135

230.30303

222.60869

204.00000

190.69767

181.33333

177.77777

71.25000

49.09090

42.35294

40.00000

40.00000

40.00000

34.18181

33.81818
Logsigtheta Sample

43

1.94224
2.02785
2.09164
2.15740
2.27181
2.40968
2.51770
2.63573
2.73869
2.87795
3.26767
3.43105
3.56372
3.54578
3.60884
3.68888
3.49926
3.46574

6.42225

6.19771

5.97289

5.86179

5.43940

5.40542

5.31812

5.25069

5.20034

5.18053

4.26619

3.89367

3.74604

3.68888

3. 68888

3.68888

3.53169

3.52100

44 

TABLE 5

Angle

179.00

178.00

177.00

176.00

175.00

174.00

173.00

172.00

171.00

170.00

160.00

150.00

140.00

130.00

120.00

110.00

100.00

90.00

179.00

178.00

177.00

176.00

175.00

174.00

173.00

172.00

171.00

170.00

160.00

150.00

140.00

130.00

120.00

110.00

100.00

90.00
Sigmatheta

18

84.68336

57.86113

54.77651

46.16132

35.17316

22.98136

20.00000

$19.5182 ?$

20.00000

21.54195

30.13393

12.72015

52.52101

59.52381

68.68132

74.40476

81.16883

18

89.28571

239.32253

87.78780

61.34969

46.16132

32.46753

22.04969

18.92857

18.68770

17.61905

18.70748

30.13393

40.58441

52.52101

61.90476

71.42857

77.38095

87.66234

92.85714
Logsigtheta Sample

45

4.43892

4.05805

4.00326

3.83214

3.56028

3.13468

2.99573

2. 97135

2. 99573

3.07000

3.40565

2.54319

3.96121

4.08638

4.22948

4.30952

4. 39653

4.49184

5.47781

4.47492

4.11659

3.83214

3.48024

3.09330

2.94067

2. 92787

2. 86898

2.92892

3.40565

3.70338

3.96121

4.12560

4.26870

4. 34874

4.47349

4. 53106 

APPENDIX II

ILIUSTRATIONS

65 



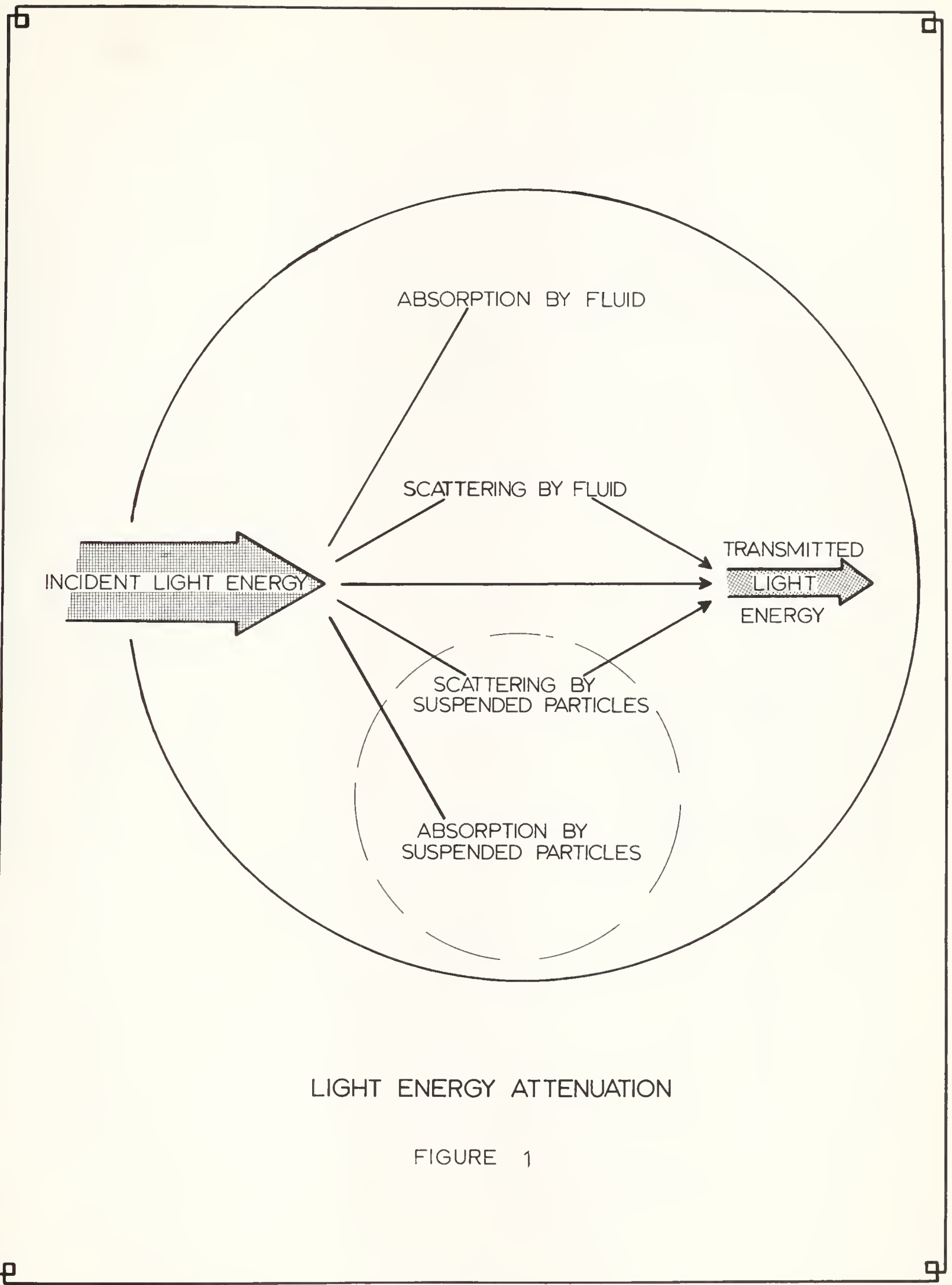





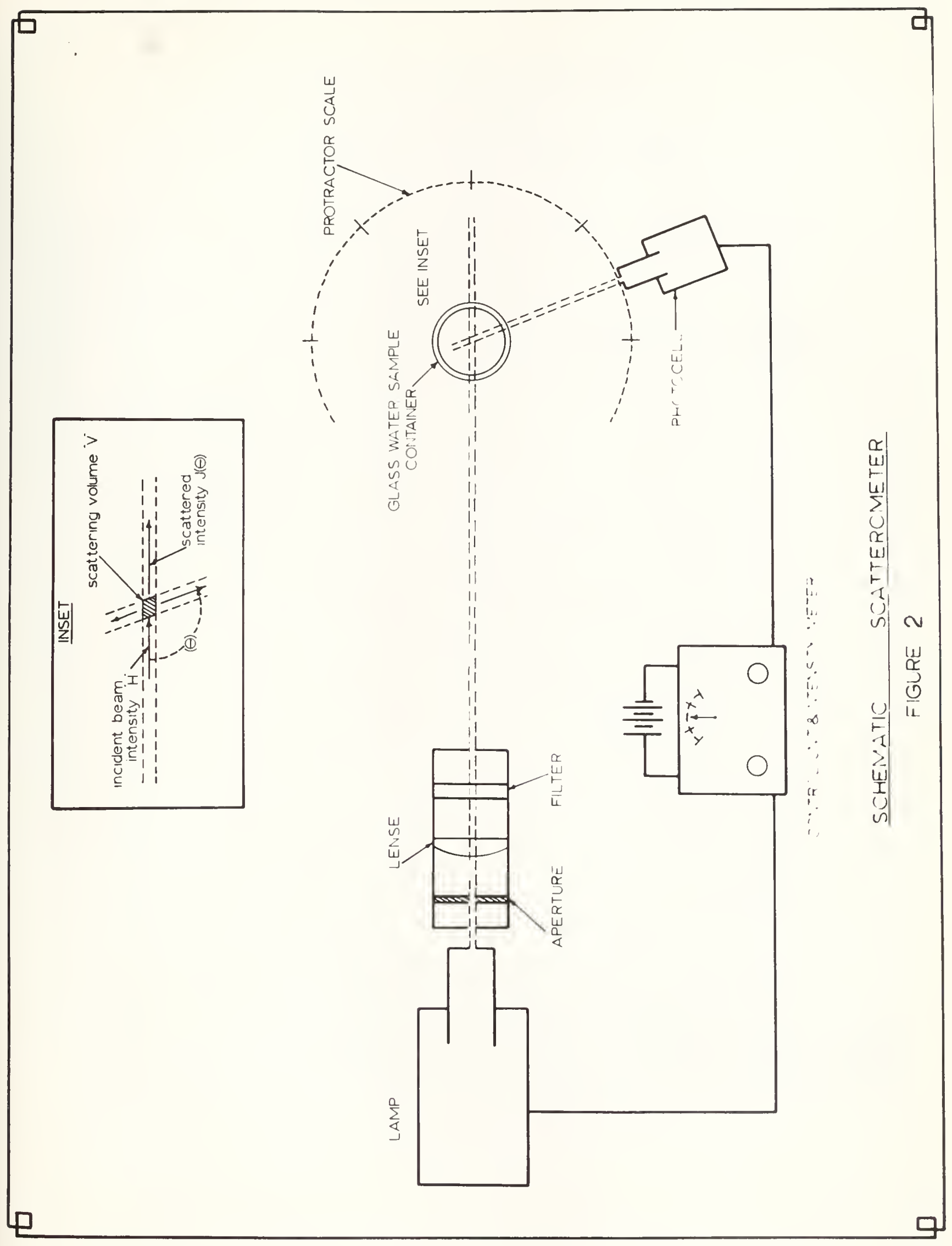





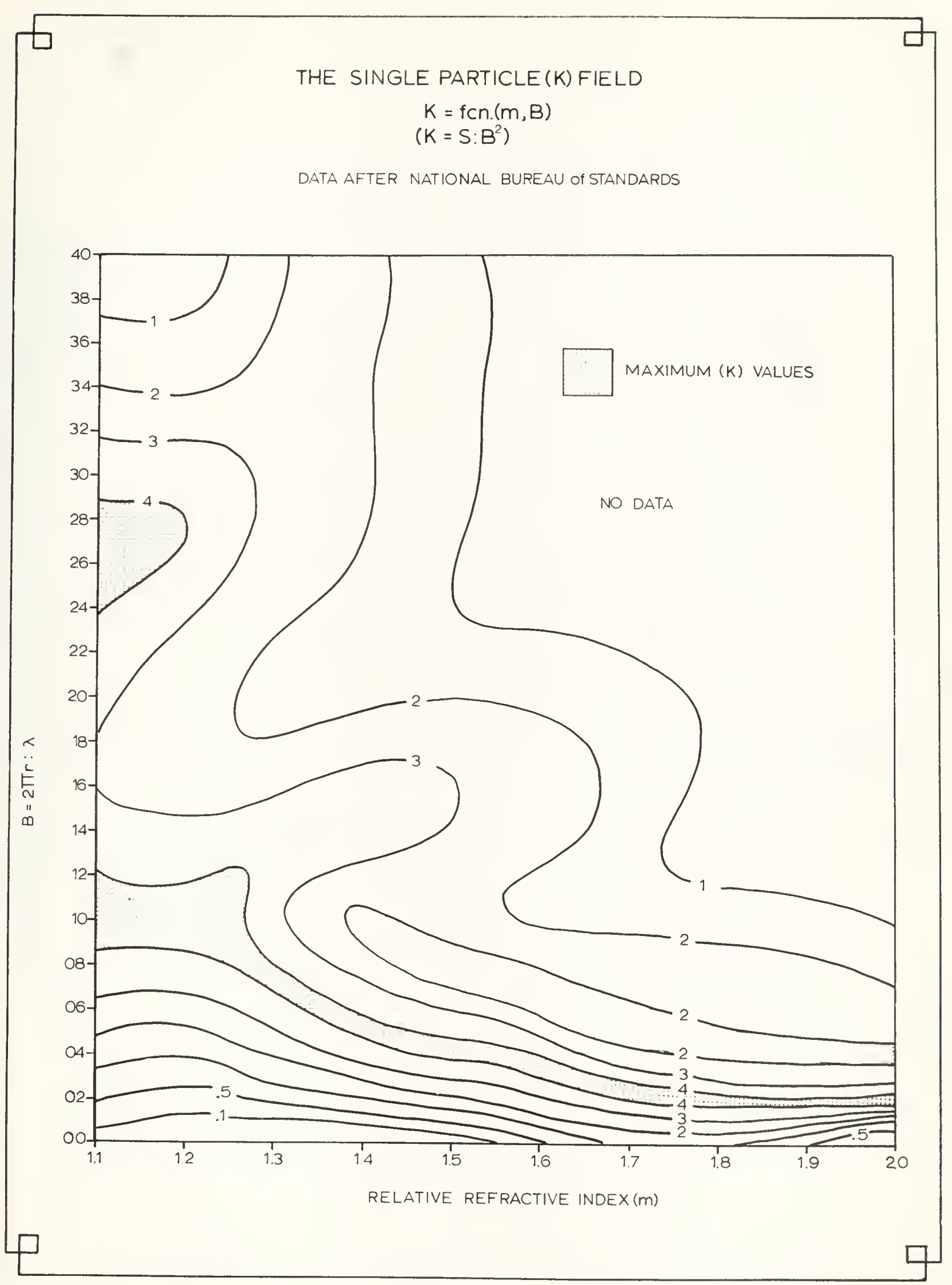

FIGURE 3 



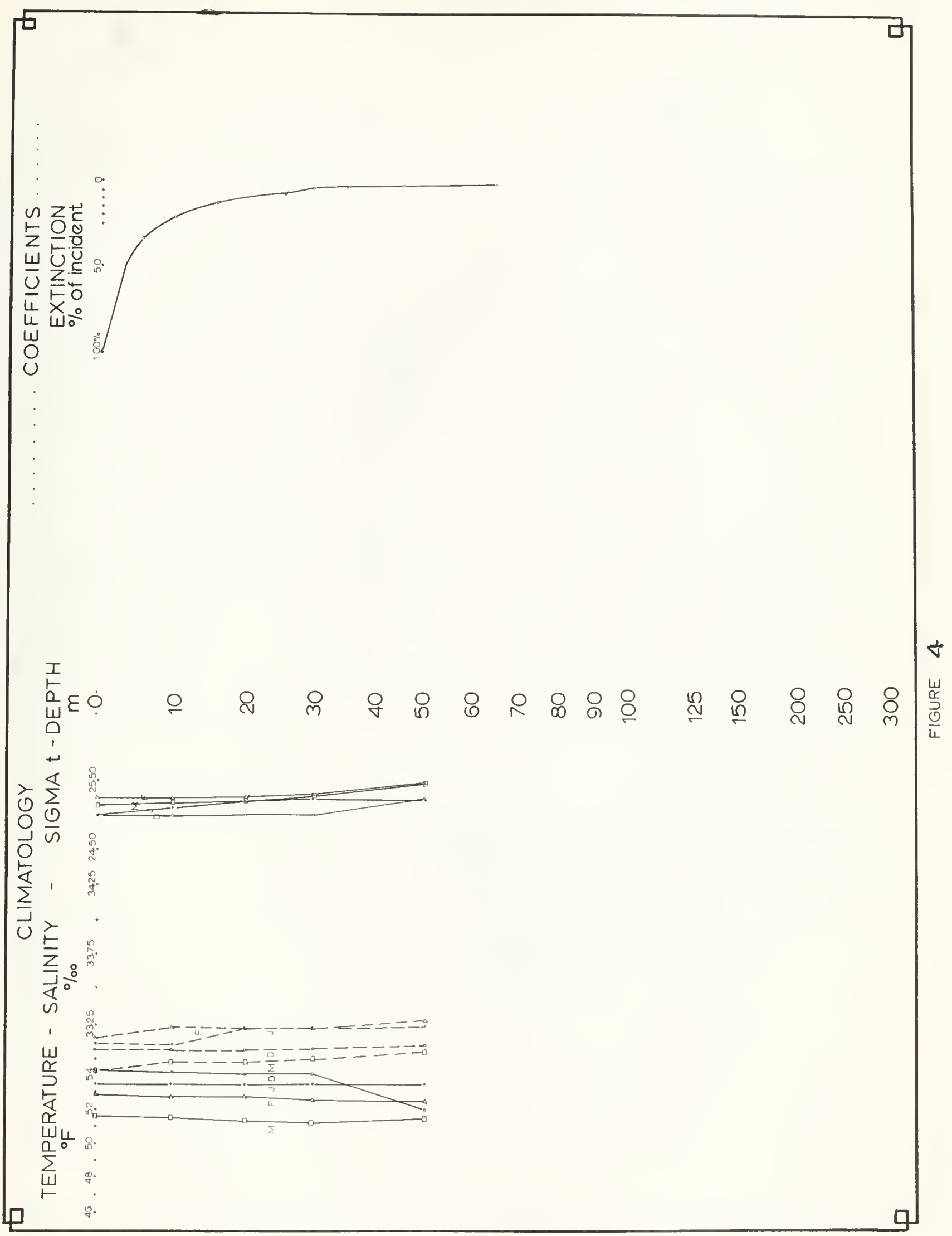





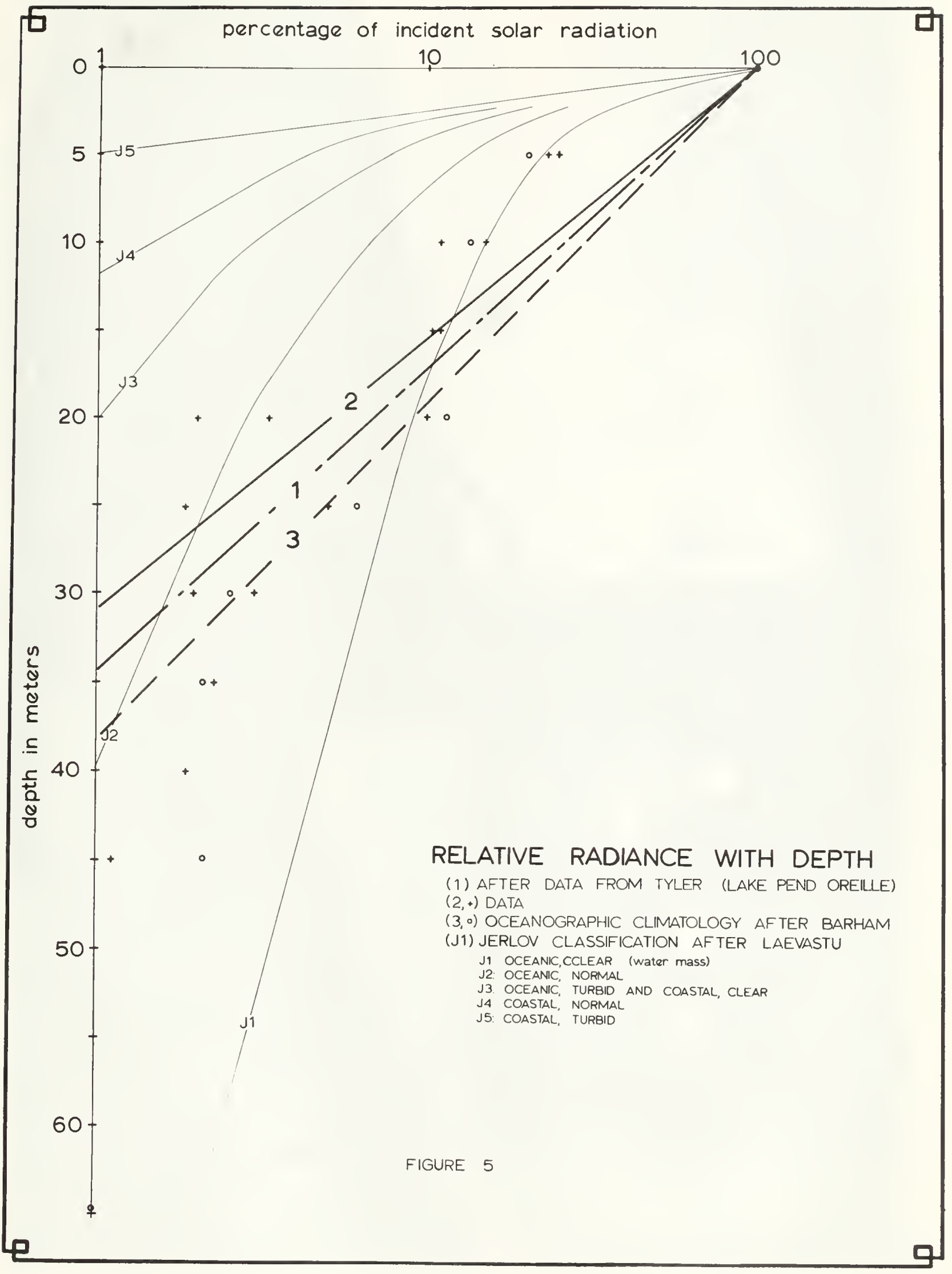





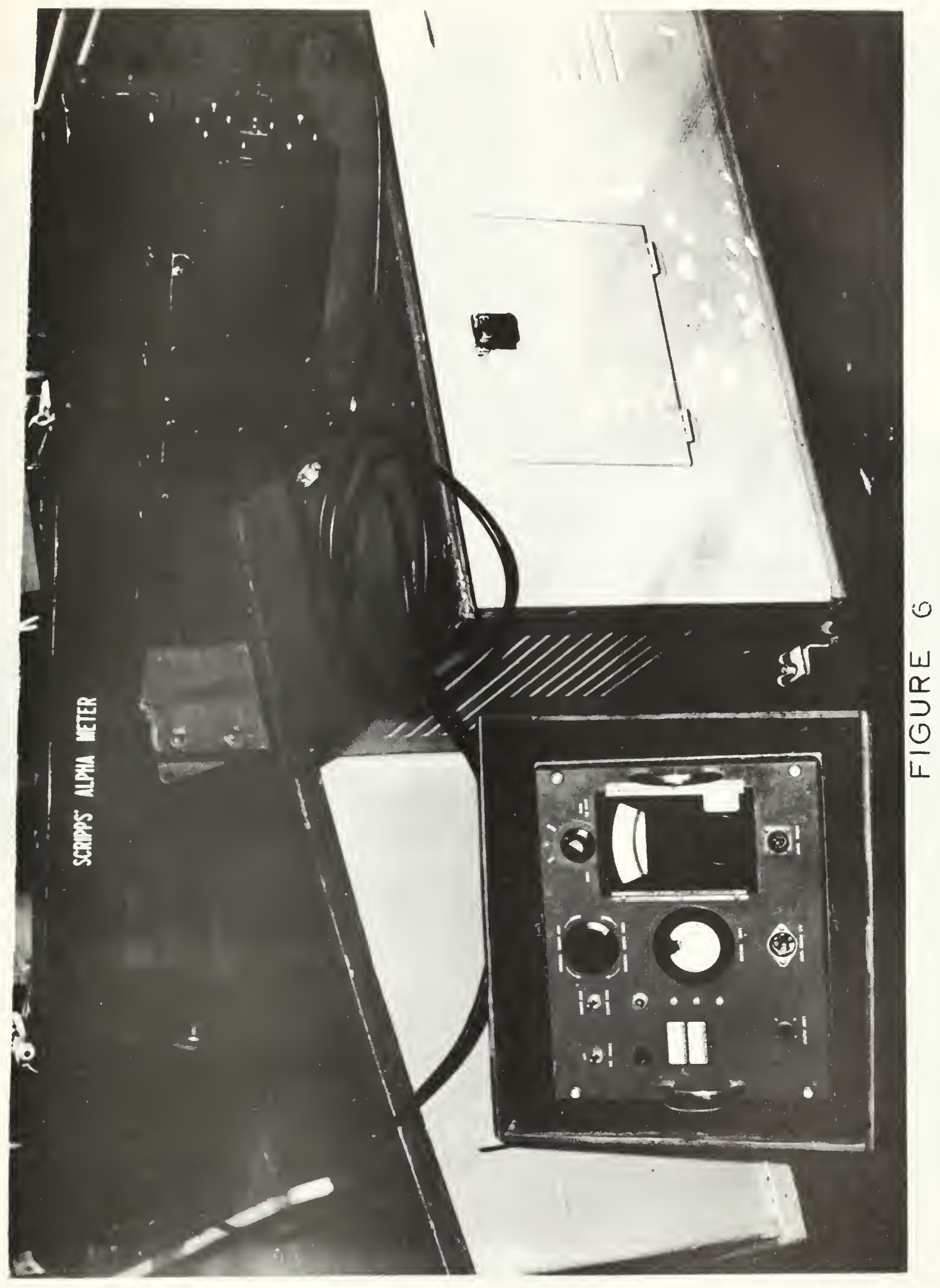





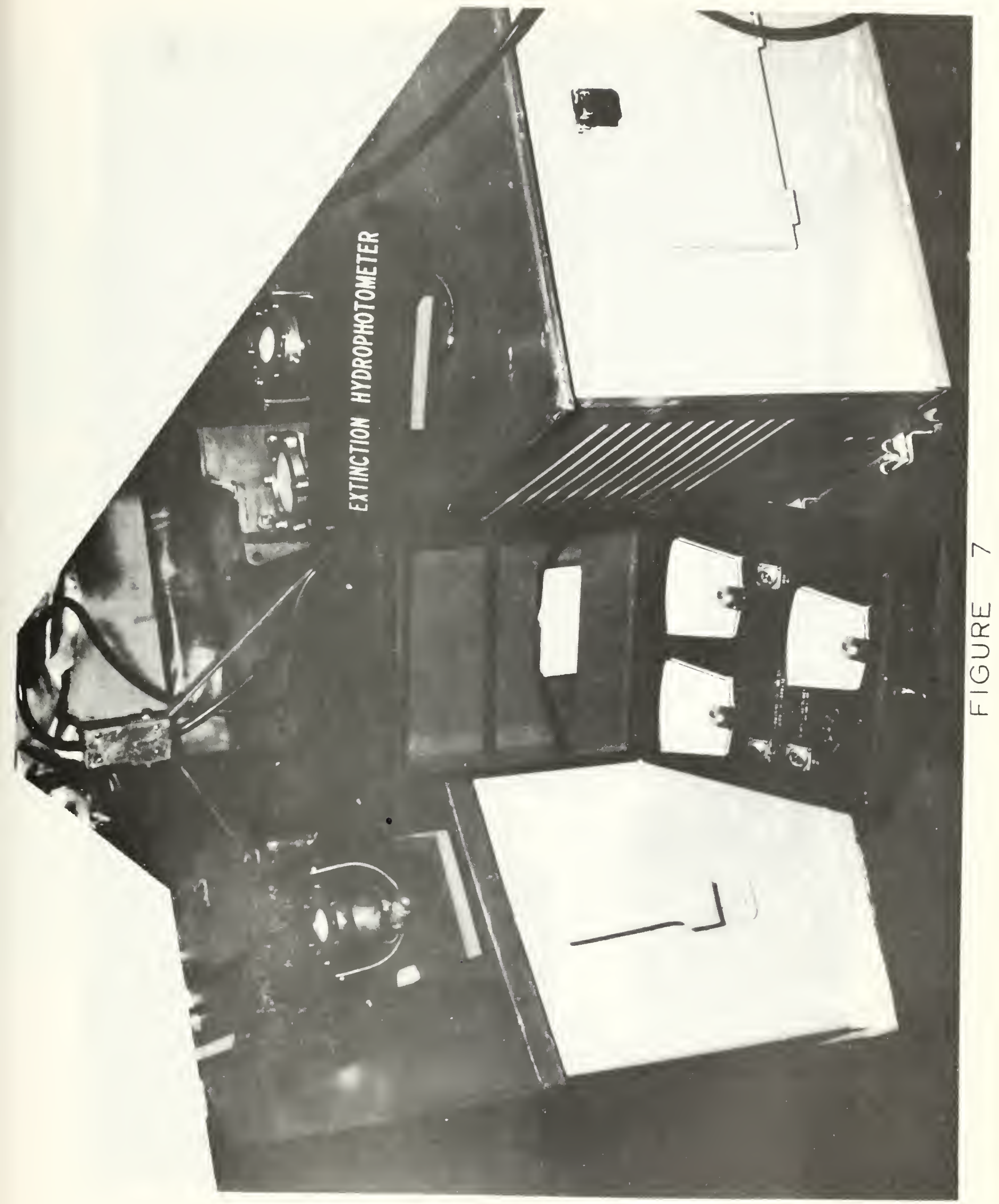





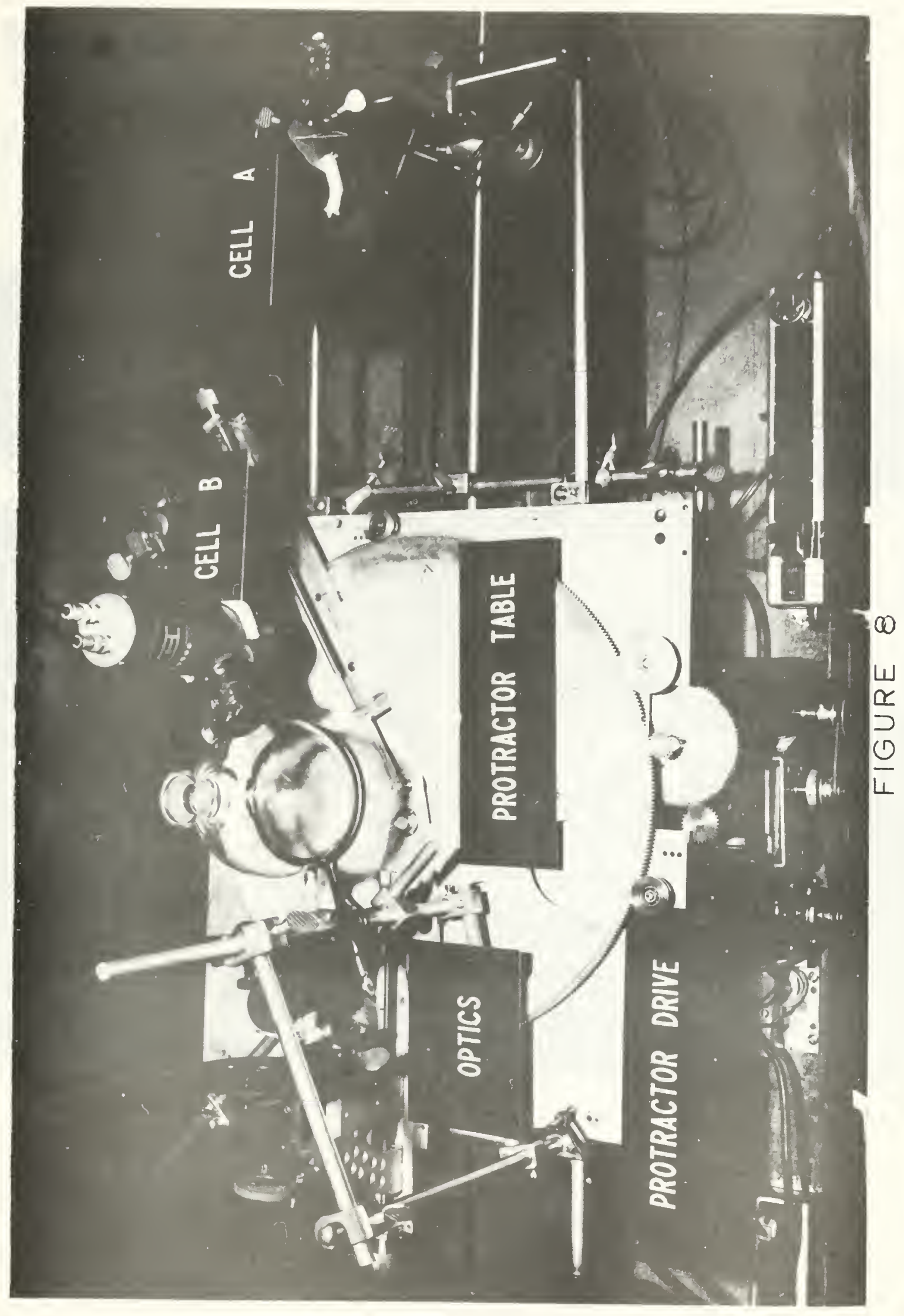





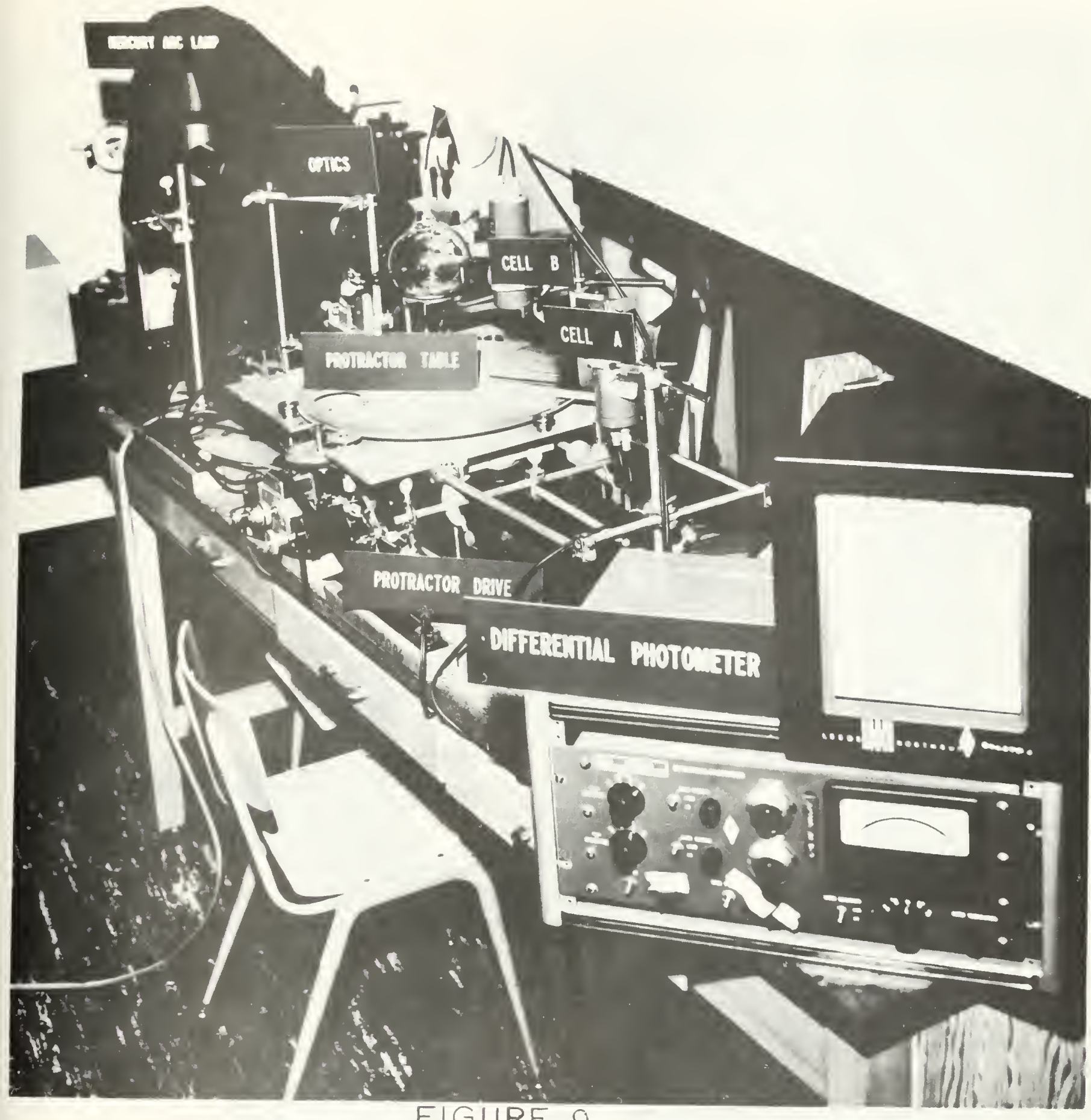

FIGURE S 



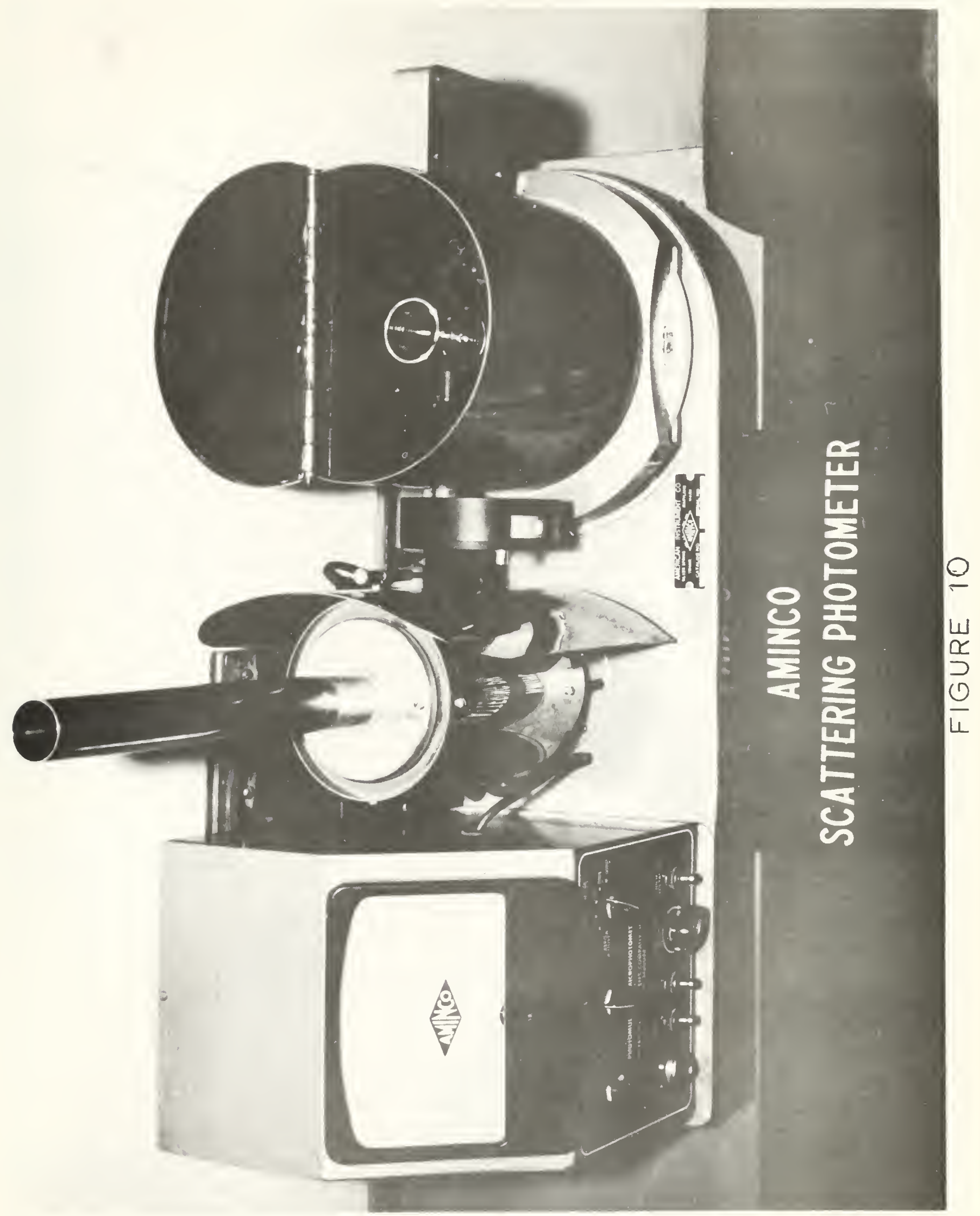





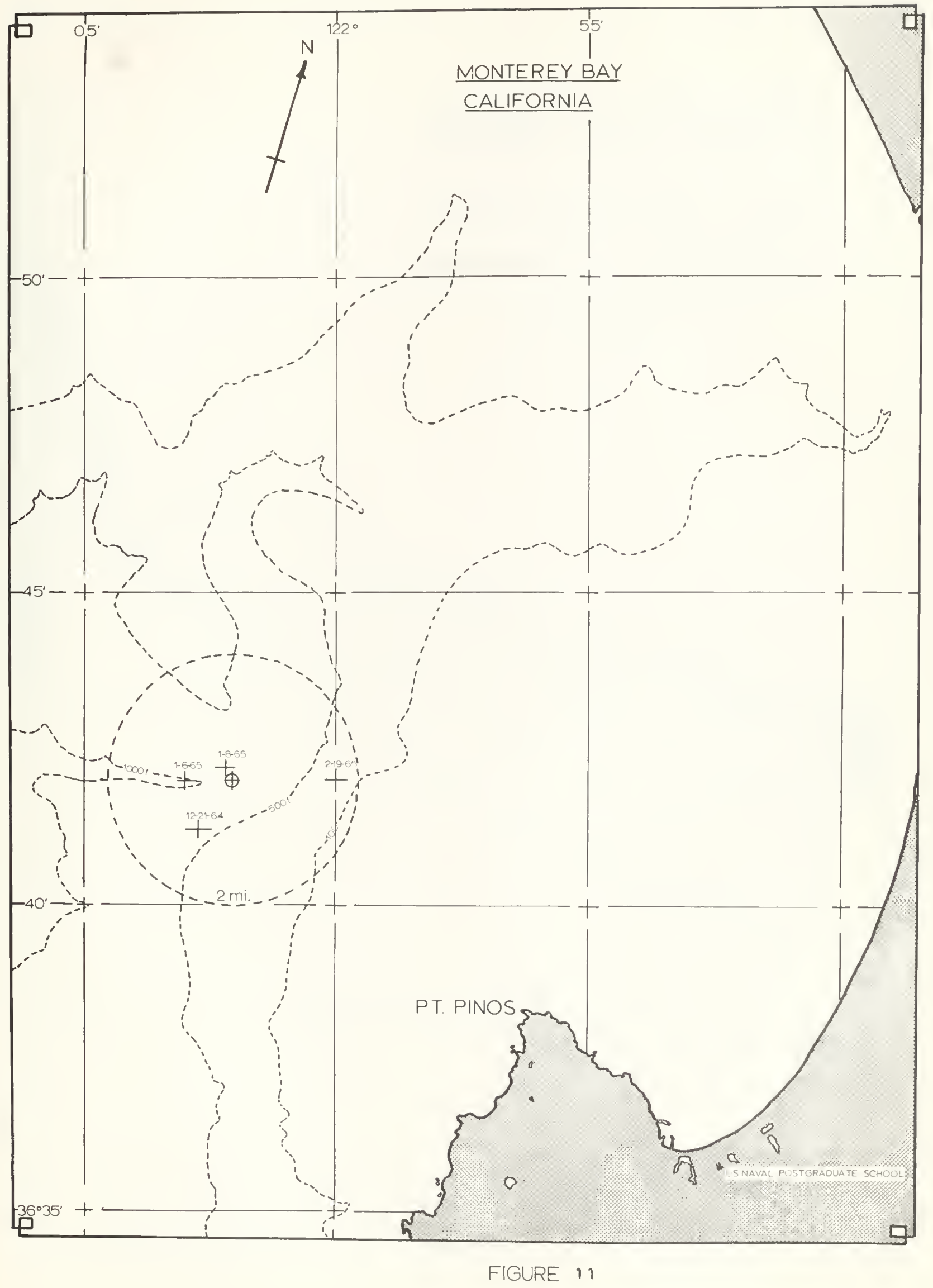





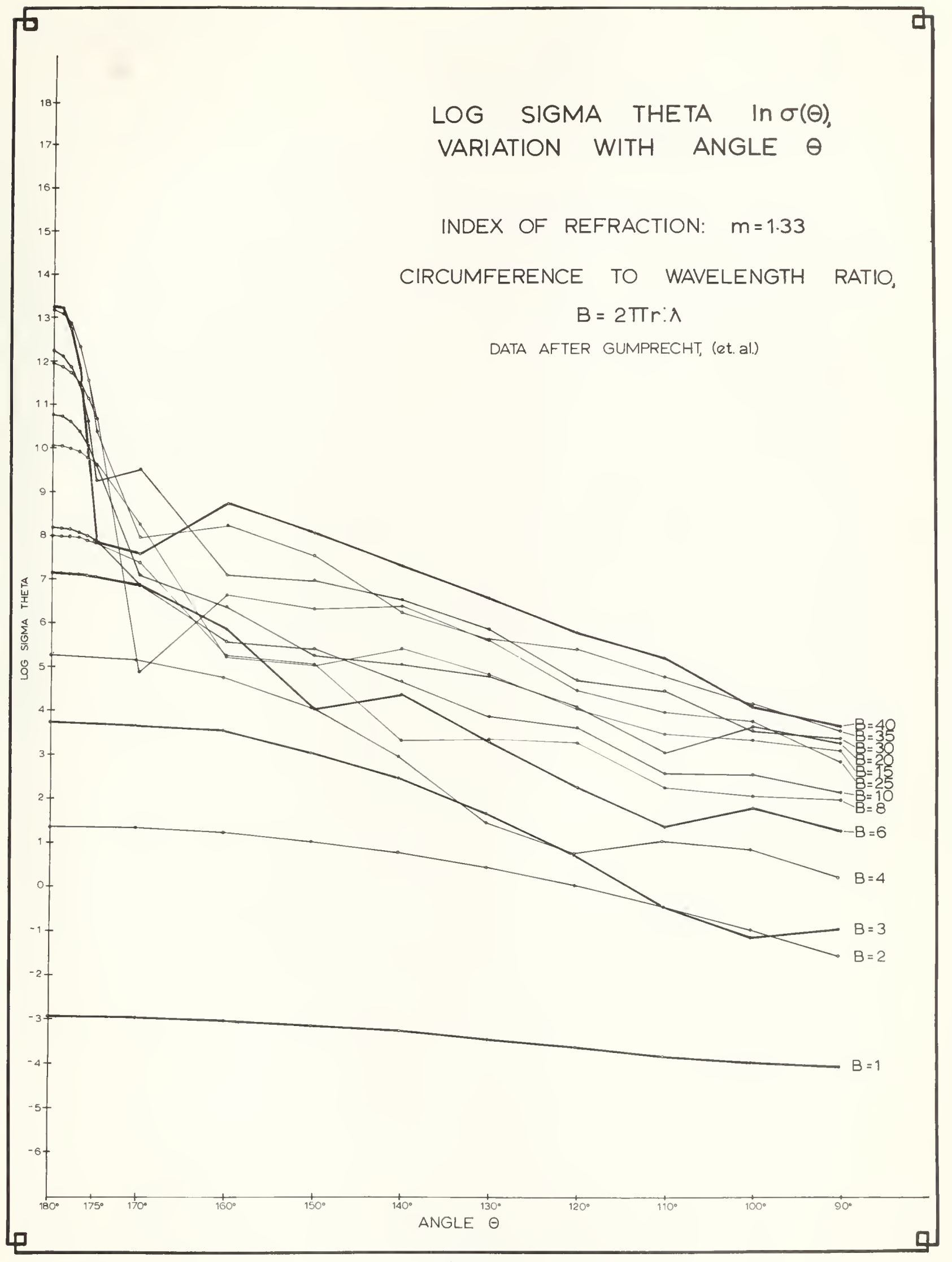

FIGURE 12 



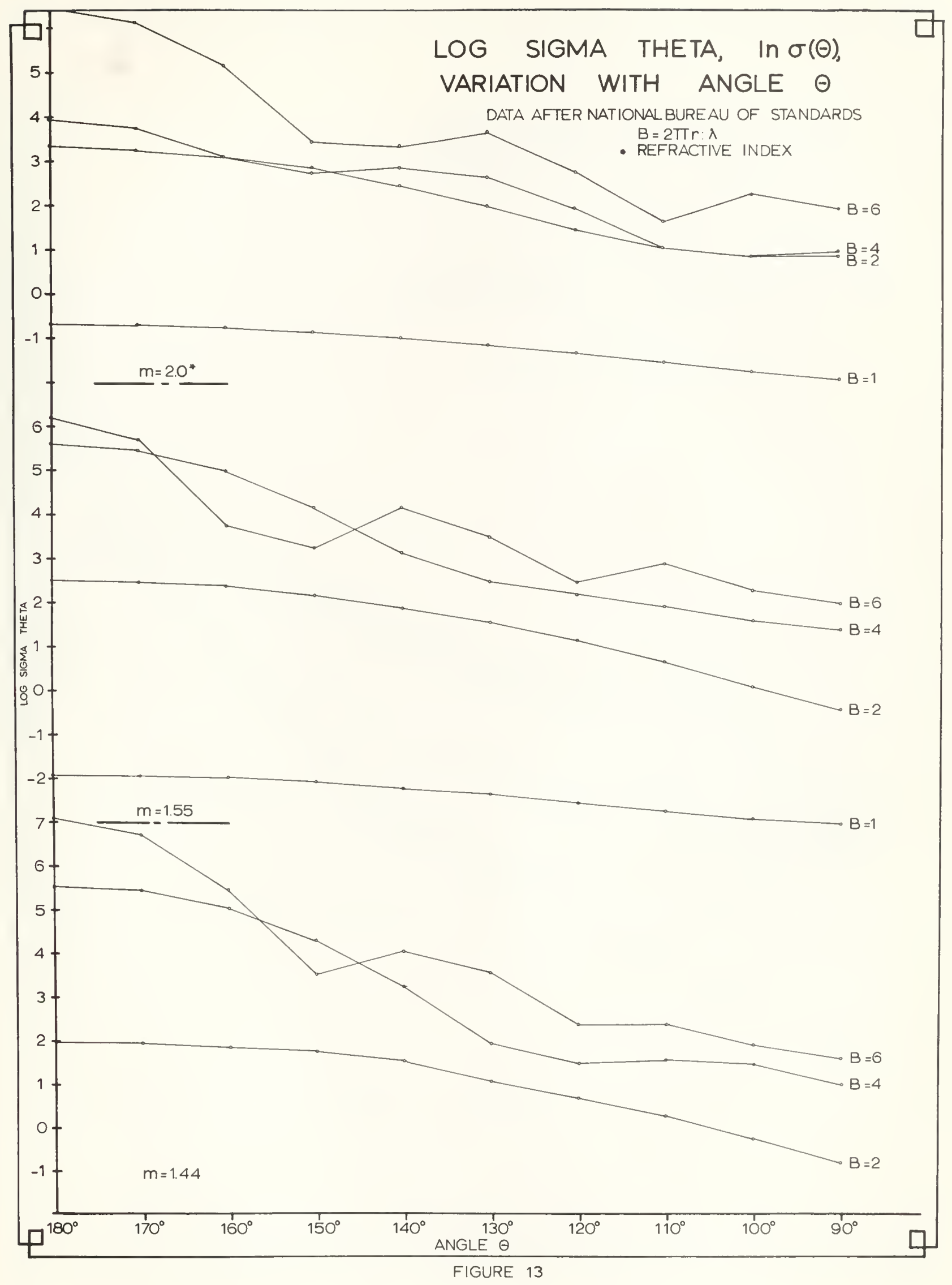





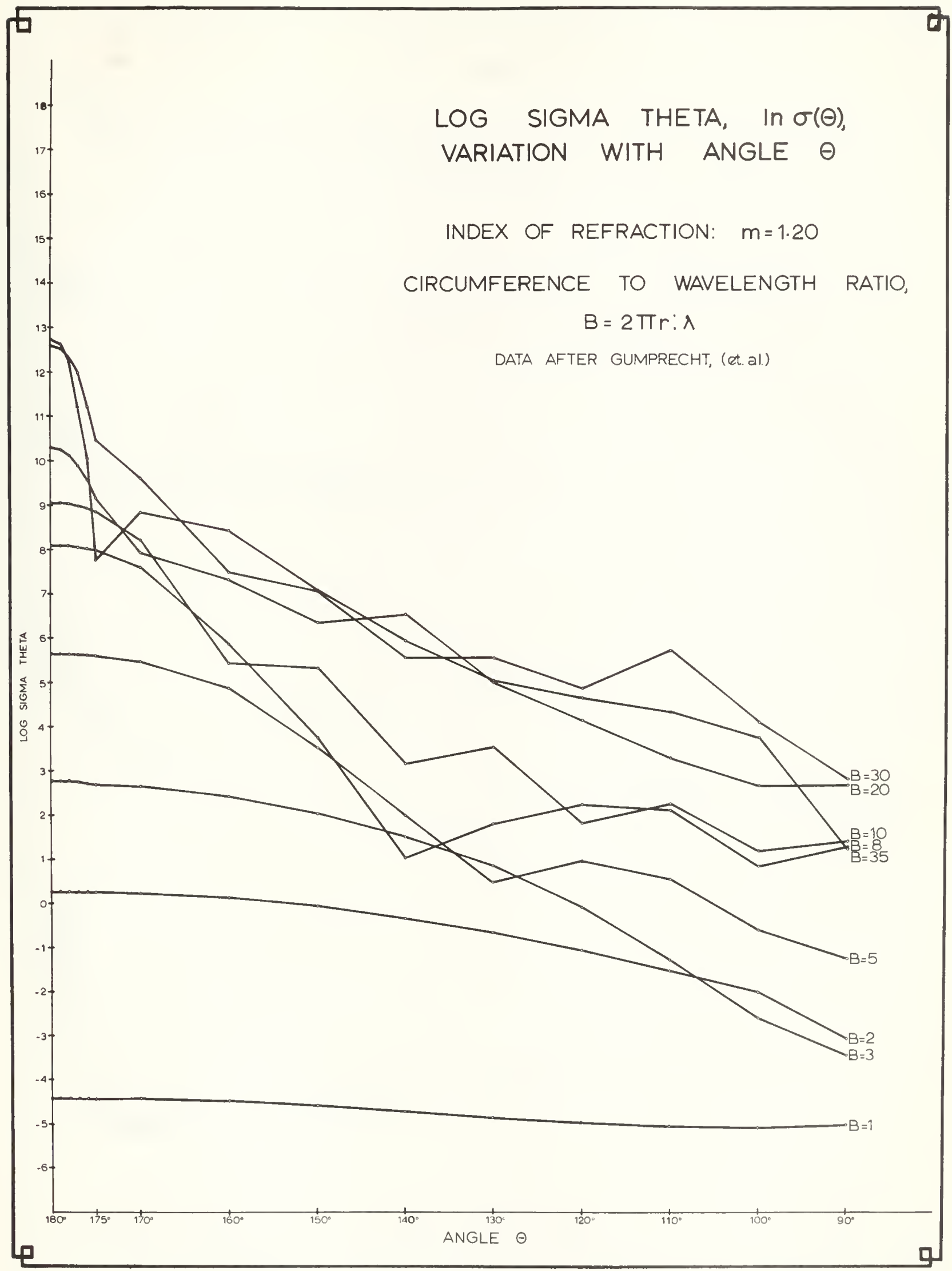

FIGURE 14 



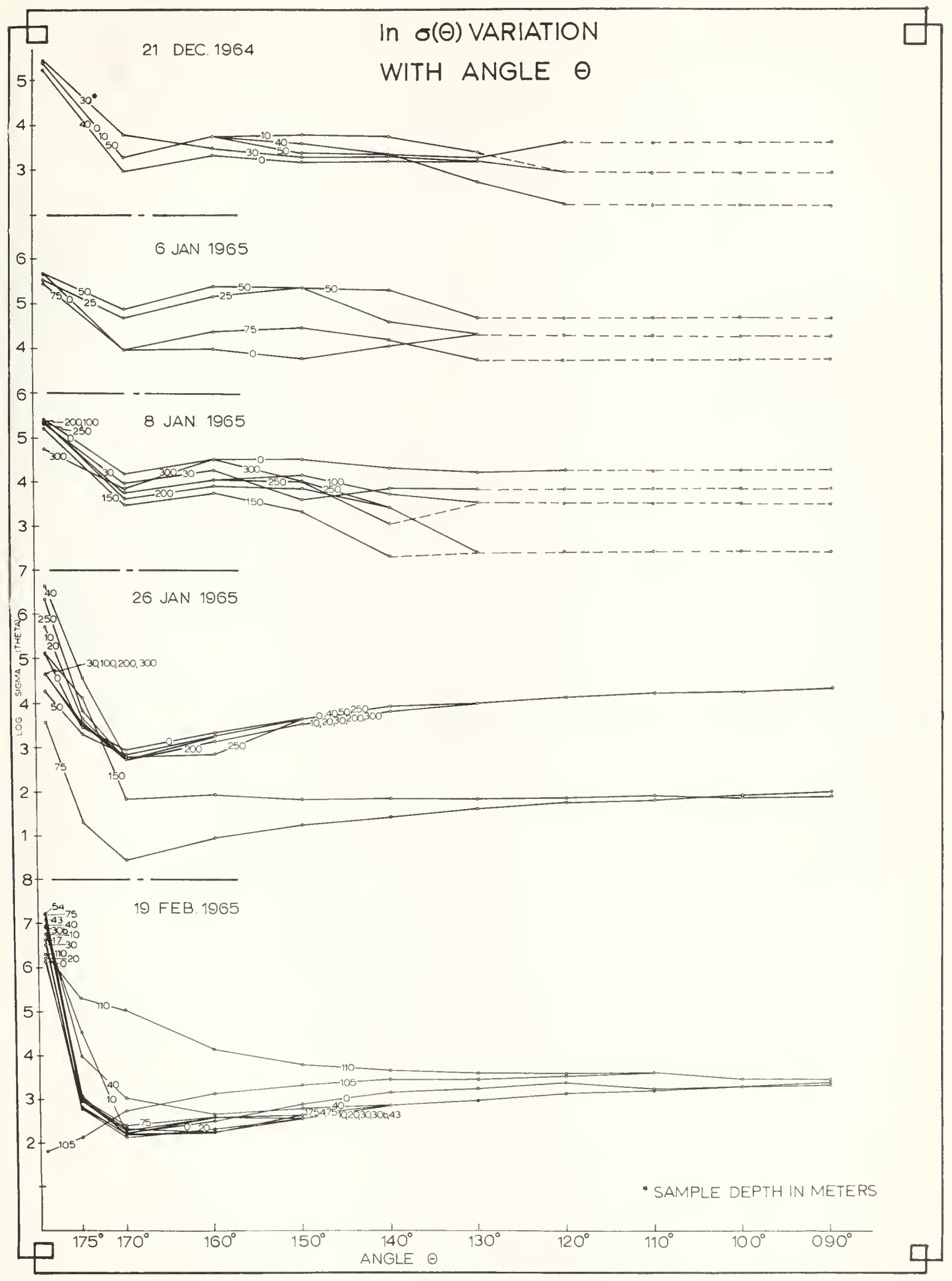

FIGURE 15 



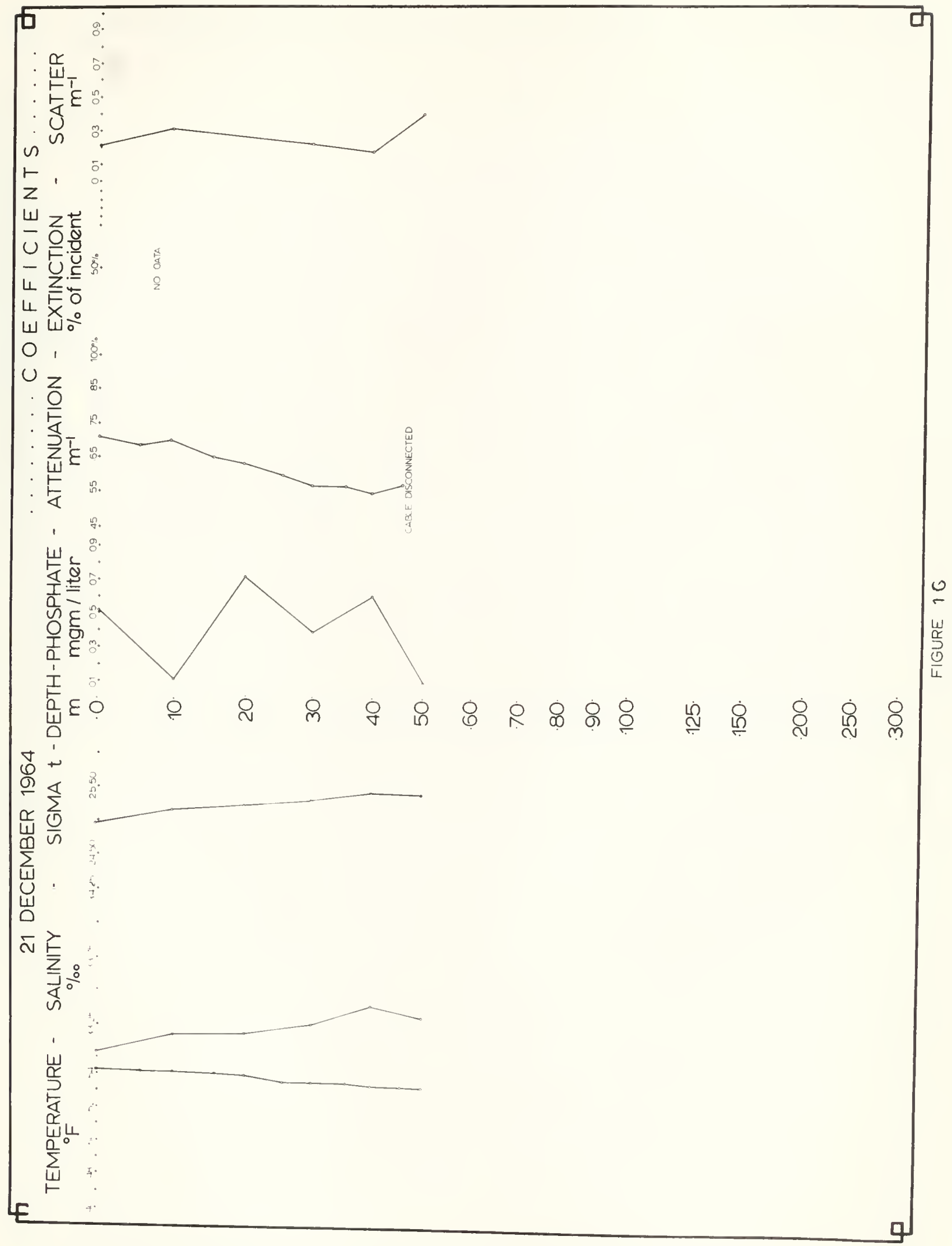





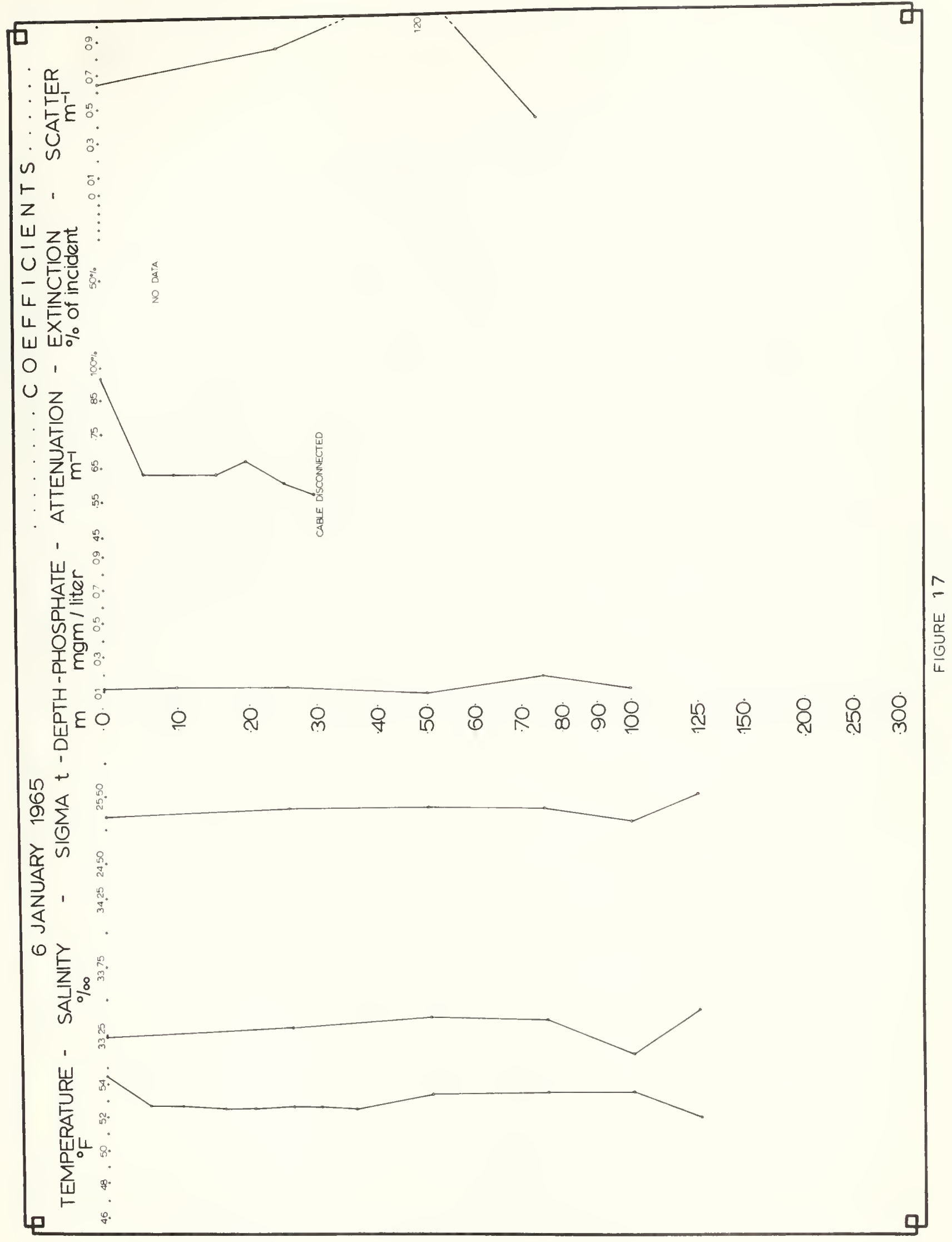





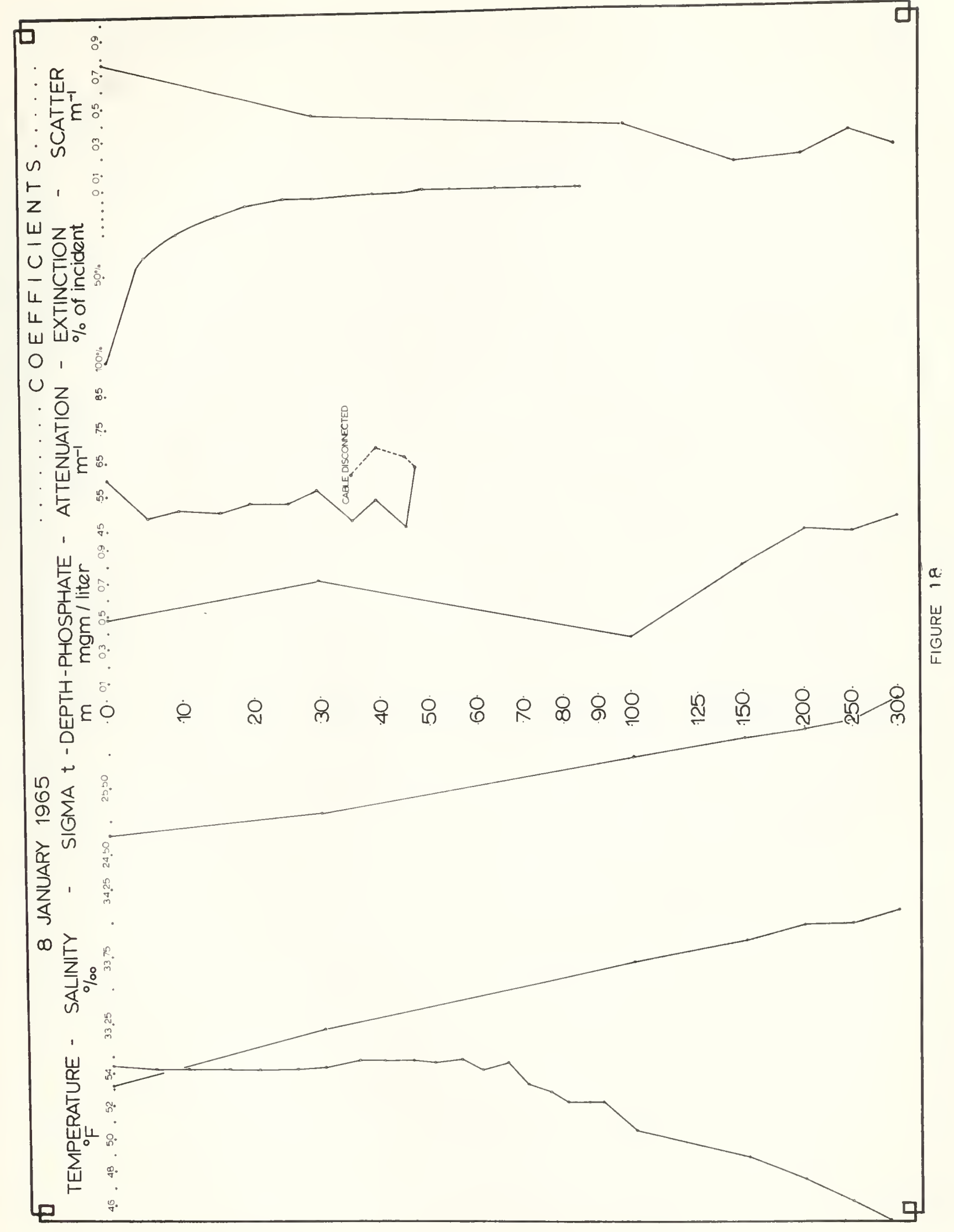





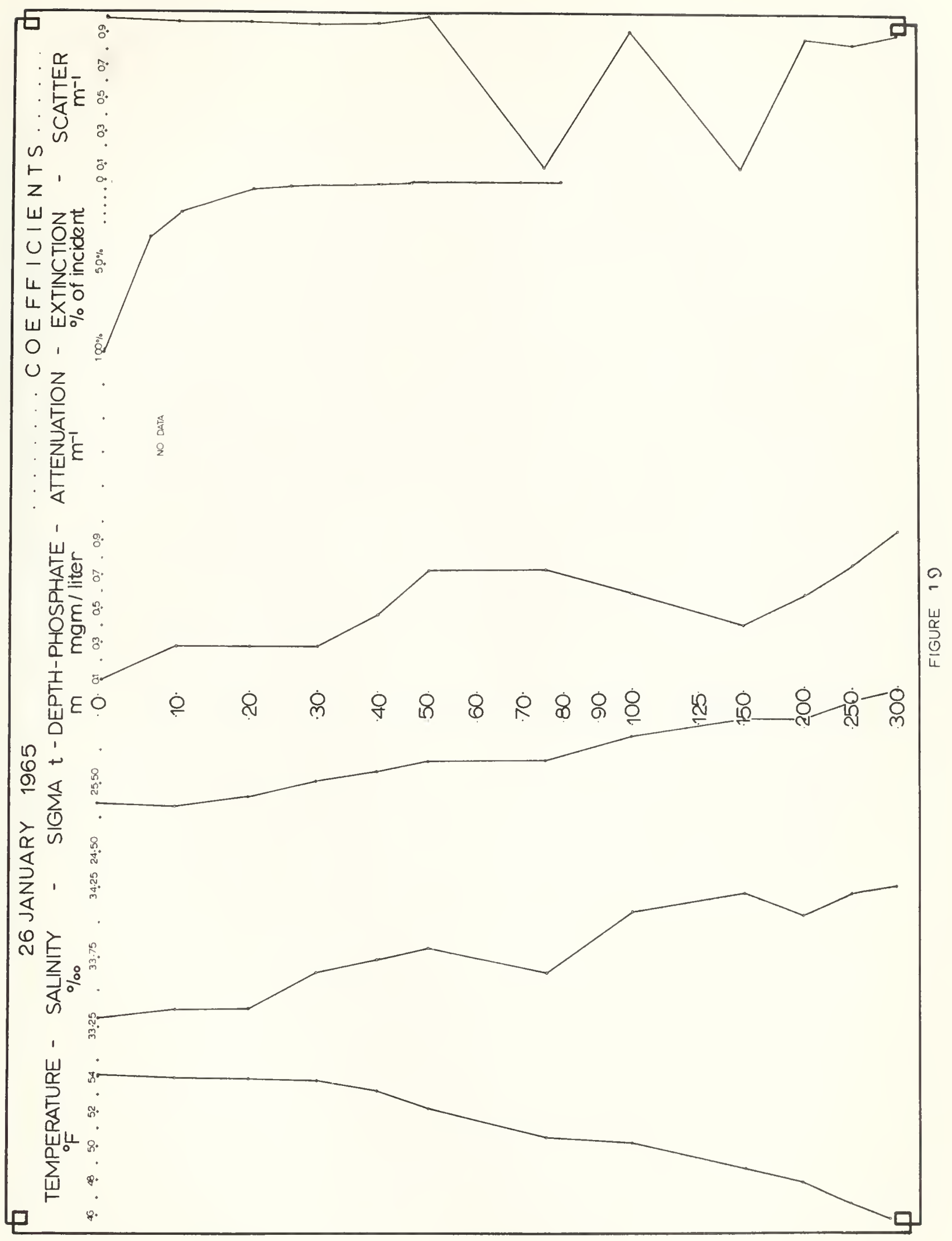





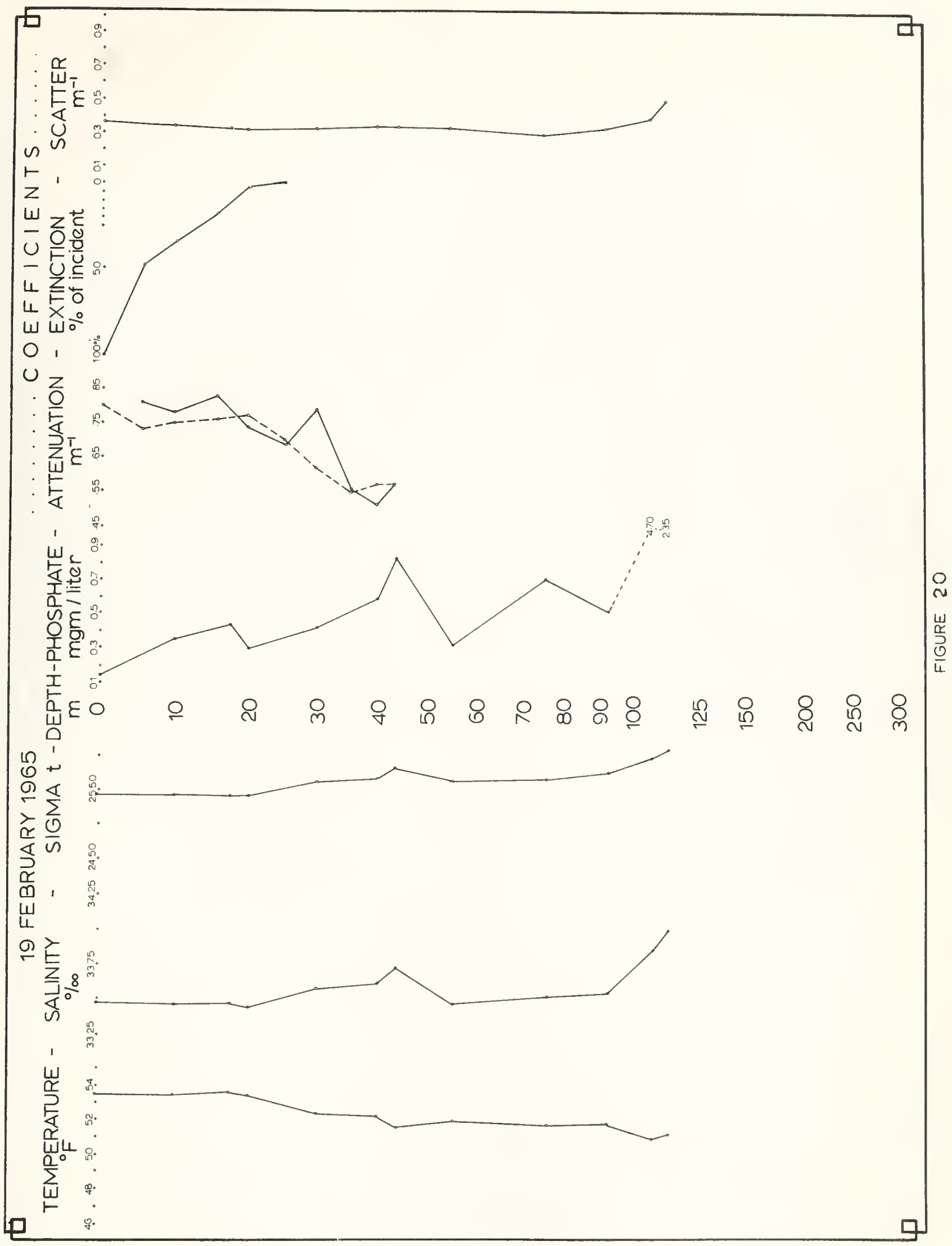





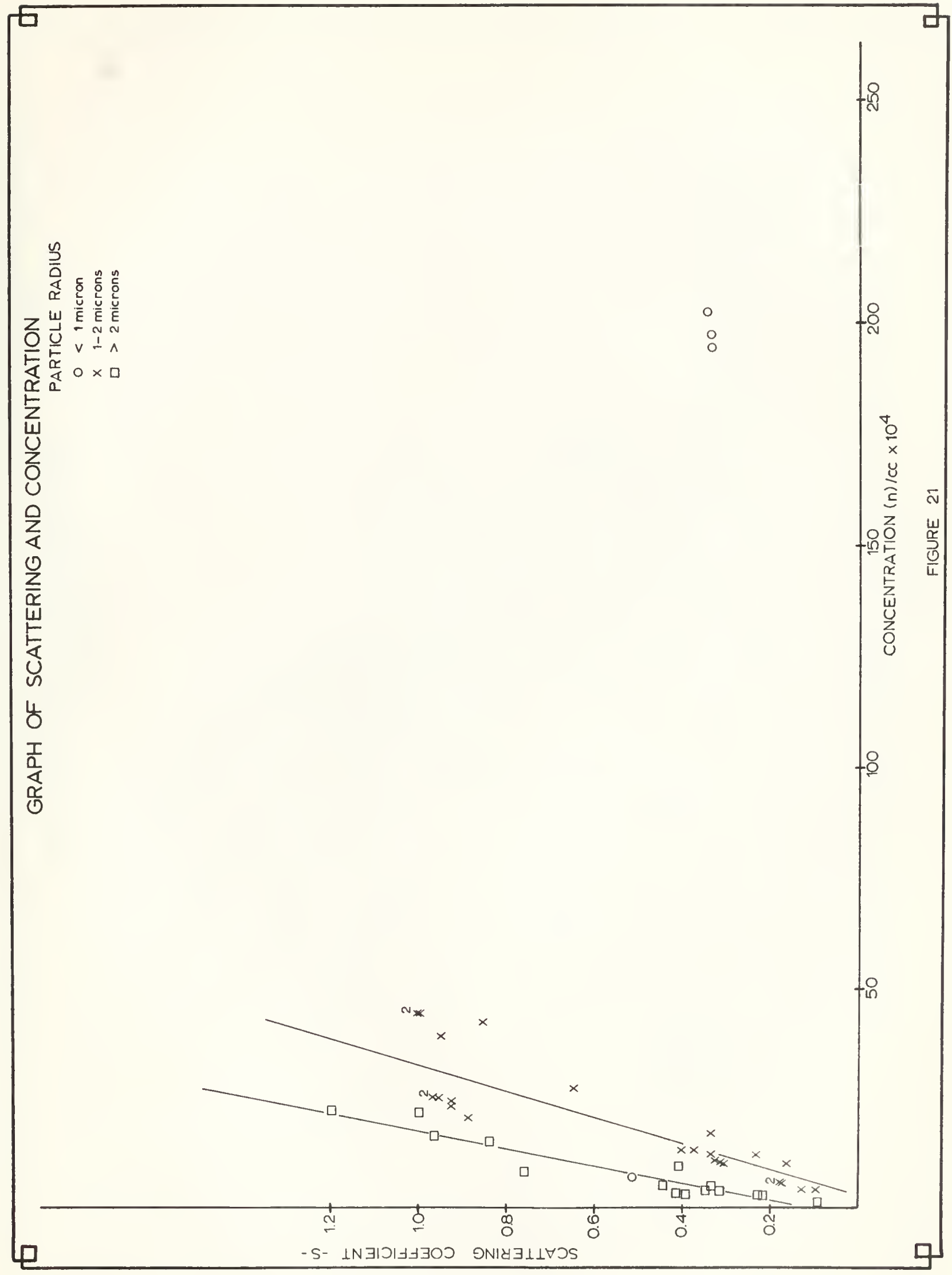





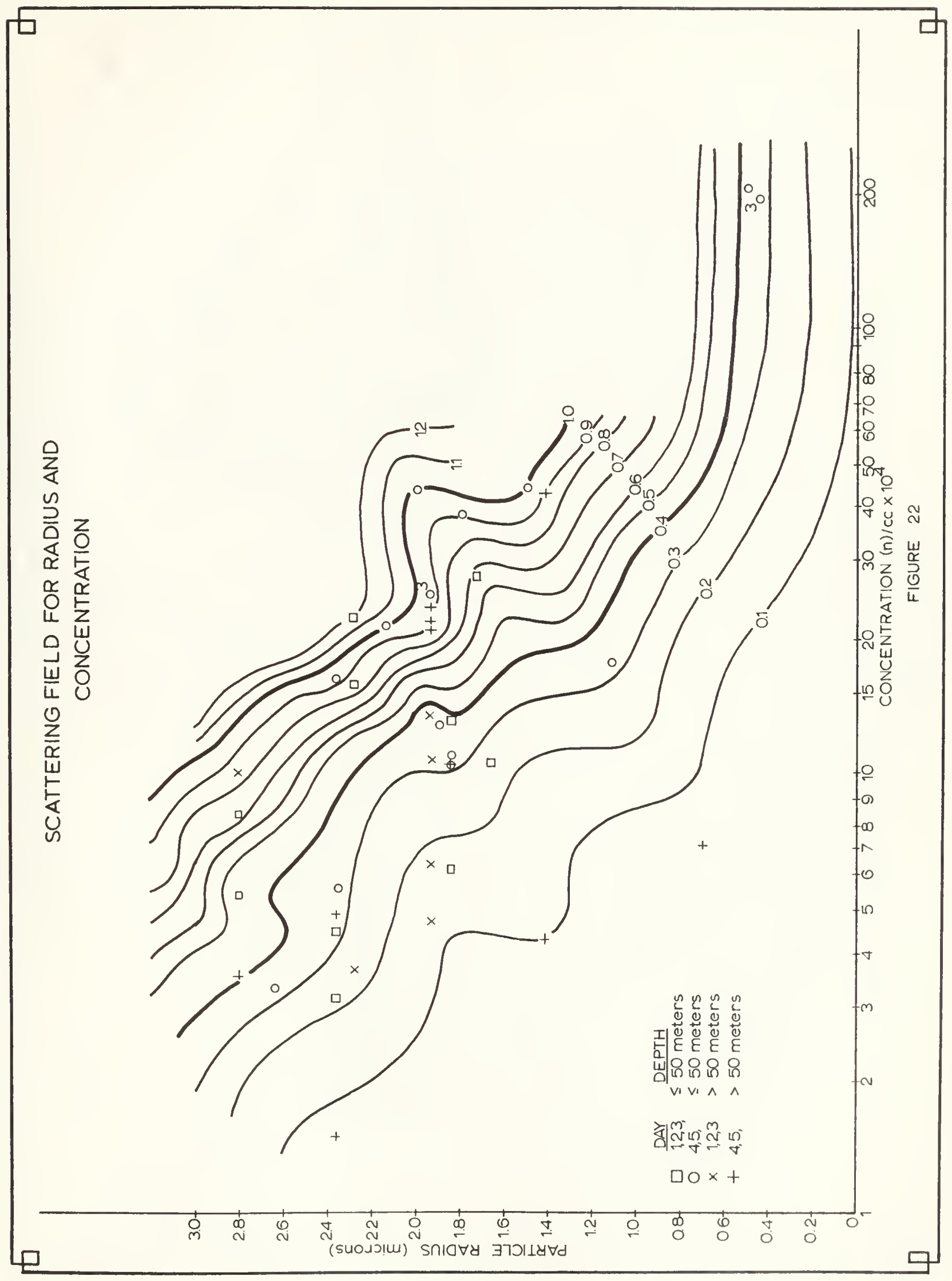





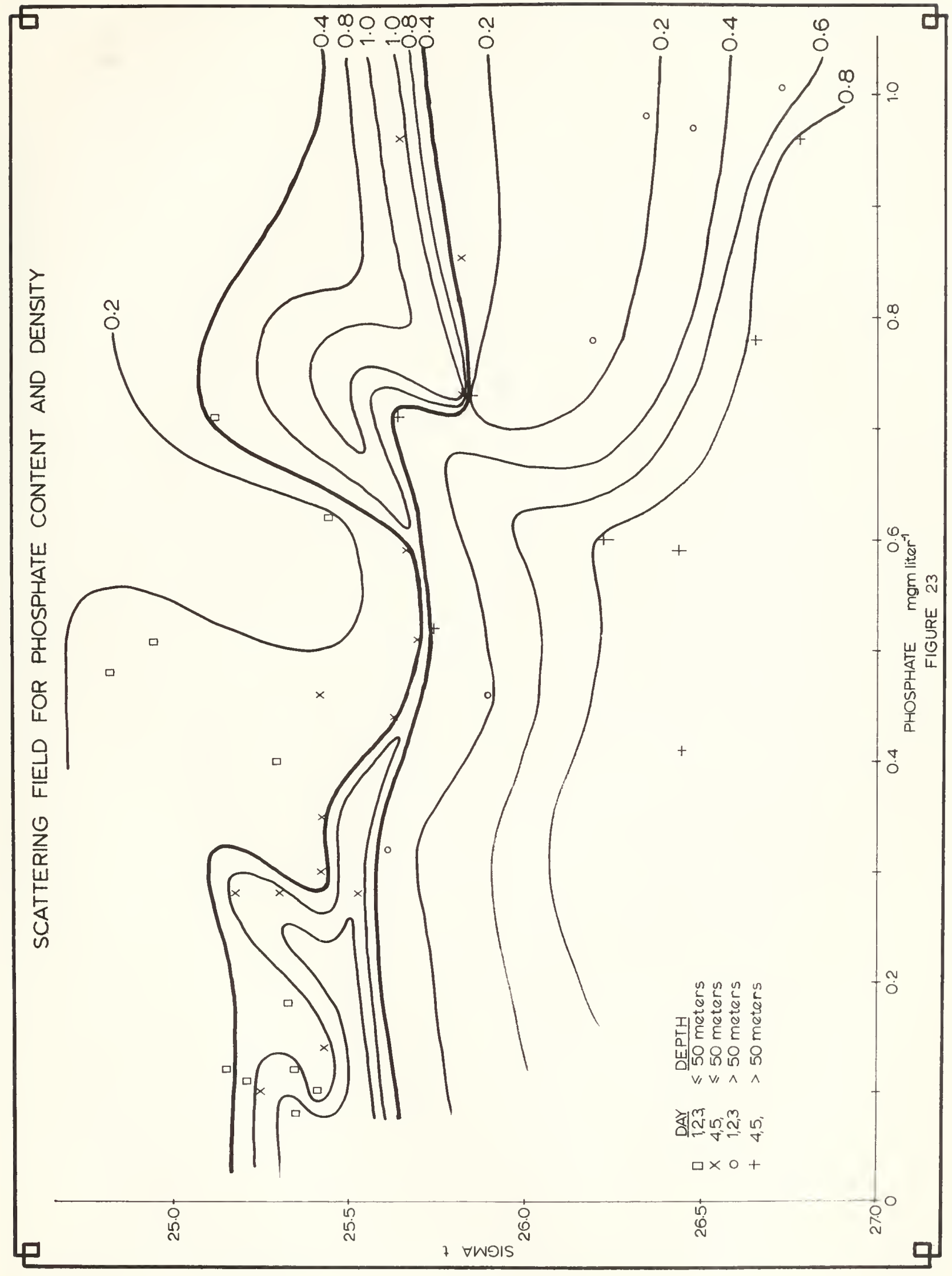







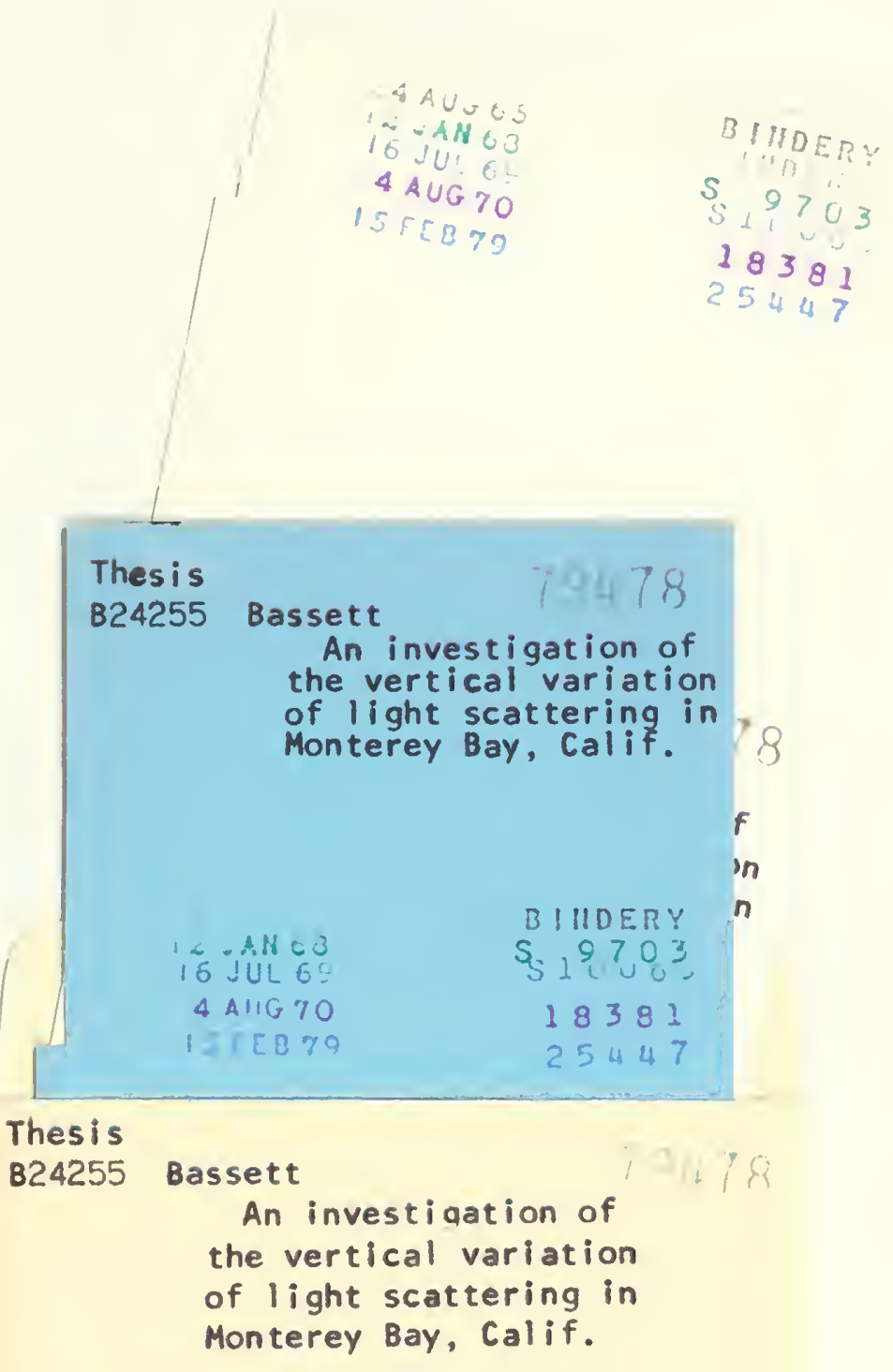


